\title{
G345.45+1.50: an expanding ring-like structure with massive star formation $\star, \star \star$
}

\author{
Cristian López-Calderón ${ }^{1,2,3}$, Leonardo Bronfman ${ }^{1}$, Lars-Åke Nyman ${ }^{2,4}$, Guido Garay ${ }^{1}$, \\ Itziar de Gregorio-Monsalvo ${ }^{2,4,5}$, and Per Bergman ${ }^{6}$
}

\footnotetext{
1 Departamento de Astronomía, Universidad de Chile, Casilla 36-D, Santiago, Chile e-mail: clopez@das.uchile.cl

2 Joint ALMA Observatory (JAO), Alonso de Córdova 3107, Vitacura, Santiago, Chile

3 National Radio Astronomy Observatory, Charlottesville, VA 22903, USA

4 European Southern Observatory, Alonso de Córdova 3107, Vitacura, Santiago, Chile

5 ESO Garching, Karl-Schwarzschild Str. 2, 85748 Garching, Germany

${ }^{6}$ Onsala Space Observatory, Chalmers Univ. of Technology, 43992 Onsala, Sweden
}

Received 14 April 2013 / Accepted 5 July 2016

\begin{abstract}
Context. Ring-like structures in the interstellar medium (ISM) are commonly associated with high-mass stars. Kinematic studies of large structures in giant molecular clouds (GMCs) toward these ring-like structures may help us to understand how massive stars form.

Aims. The origin and properties of the ring-like structure G345.45+1.50 is investigated through observations of the ${ }^{13} \mathrm{CO}(3-2)$ line. The aim of the observations is to determine the kinematics in the region and to compare physical characteristics estimated from gas emission with those previously determined using dust continuum emission. This area in the sky is well suited for studies like this because the ring is located 1.5 above the Galactic plane at $1.8 \mathrm{kpc}$ from the Sun, thus molecular structures are rarely superposed on our line of sight, which minimizes confusion effects that might hinder identifying of individual molecular condensations.

Methods. The ${ }^{13} \mathrm{CO}(3-2)$ line was mapped toward the whole ring using the Atacama Pathfinder Experiment (APEX) telescope. The observations cover $17^{\prime} \times 20^{\prime}$ in the sky with a spatial resolution of $0.2 \mathrm{pc}$ and an rms of $\sim 1 \mathrm{~K}$ at a spectral resolution of $0.1 \mathrm{~km} \mathrm{~s}^{-1}$. Results. The ring is found to be expanding with a velocity of $1.0 \mathrm{~km} \mathrm{~s}^{-1}$, containing a total mass of $6.9 \times 10^{3} M_{\odot}$, which agrees well with that determined using $1.2 \mathrm{~mm}$ dust continuum emission. An expansion timescale of $\sim 3 \times 10^{6} \mathrm{yr}$ and a total energy of $\sim 7 \times 10^{46} \mathrm{erg}$ are estimated. The origin of the ring might have been a supernova explosion, since a $35.5 \mathrm{~cm}$ source, J165920-400424, is located at the center of the ring without an infrared counterpart. The ring is fragmented, and 104 clumps were identified with diameters of between 0.3 and $1.6 \mathrm{pc}$, masses of between 2.3 and $7.5 \times 10^{2} M_{\odot}$, and densities of between $\sim 10^{2}$ and $\sim 10^{4} \mathrm{~cm}^{-3}$. At least $18 \%$ of the clumps are forming stars, as is shown in infrared images. Assuming that the clumps can be modeled as Bonnor-Ebert spheres, 13 clumps are collapsing, and the rest of them are in hydrostatic equilibrium with an external pressure with a median value of $4 \times 10^{4} \mathrm{~K} \mathrm{~cm}^{-3}$. In the region, the molecular outflow IRAS 16562-3959 is identified, with a velocity range of $38.4 \mathrm{~km} \mathrm{~s}^{-1}$, total mass of $13 M_{\odot}$, and kinematic energy of $7 \times 10^{45} \mathrm{erg}$. Finally, five filamentary structures were found at the edge of the ring with an average size of $3 \mathrm{pc}$, a width of $0.6 \mathrm{pc}$, a mass of $2 \times 10^{2} M_{\odot}$, and a column density of $6 \times 10^{21} \mathrm{~cm}^{-2}$.
\end{abstract}

Key words. ISM: clouds - stars: massive - stars: formation - dust, extinction - ISM: kinematics and dynamics - ISM: molecules

\section{Introduction}

A wealth of observations have shown that ring- and shell-like structures in the interstellar medium (ISM) are ubiquitous (e.g. Churchwell et al. 2006, 2007; Churchwell 2008; Heiles 1979; Martín-Pintado et al. 1999; Oka et al. 1998; Schuller et al. 2009; Wong et al. 2008; Beaumont \& Williams 2010; Sidorin et al. 2014; Anderson et al. 2015). In the Galaxy, these structures range up to $1.2 \mathrm{kpc}$ in radius, $2 \times 10^{7} M_{\odot}$ in mass, and

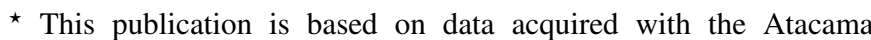
Pathfinder Experiment (APEX). APEX is a collaboration between the Max-Planck Institut für Radioastronomie, the European Southern Observatory, and the Onsala Space Observatory.

$\star \star$ The Atacama Submillimeter Telescope (ASTE) Experiment is a project driven by the National Astronomical Observatory of Japan in collaboration with Universidad de Chile, and Japanese institutes including University of Tokyo, Nagoya University, Osaka-Prefecture University, Ibaragi University, and Hokkaido University.
$10^{53} \mathrm{erg}$ in kinetic energy. Their formation has been associated with the energy released by massive stars through stellar winds, supernova explosions, and ionizing radiation, or by collisions of high-velocity HI clouds with the Galactic disk (Tenorio-Tagle \& Bodenheimer 1988). Rings with a size smaller than $100 \mathrm{pc}$ have been related with $\mathrm{OB}$ associations and stellar clusters in their interiors (Tenorio-Tagle \& Bodenheimer 1988), and hot cores and molecular clumps at their edges (e.g. Martín-Pintado et al. 1999; Wong et al. 2008).

In this study, the entire ring-like structure G345.45+1.50 was observed in the ${ }^{13} \mathrm{CO}(3-2)$ line to study its properties including the kinematics. The ring is located $\sim 1.5$ above the Galactic plane, so there is little contamination with foreground and background molecular structures, which are mainly concentrated toward the Galactic plane, thus minimizing confusion effects in identifying individual condensations. Properties of the ring and of the identified clumps are estimated from ${ }^{13} \mathrm{CO}(3-2)$ line observations, and compared with those found using the $1.2 \mathrm{~mm}$ 
continuum emission. The molecular structure is also compared with infrared observations, from $\mathrm{MSX}^{1}$ and Spitzer ${ }^{1}$.

This ring is located at a distance of $1.8 \mathrm{kpc}$ from the Sun, inside the GMC G345.5+1.0. This GMC is located approximately between $344^{\circ} 5$ and $346^{\circ} 5$ in Galactic longitude, between 0.2 and 2.0 in Galactic latitude, and between -33 and $-2 \mathrm{~km} \mathrm{~s}^{-1}$ in LSR velocity (Bronfman et al. 1989). The ringlike structure contains a total mass of $\sim 4.0 \times 10^{3} M_{\odot}$ estimated through $1.2 \mathrm{~mm}$ continuum emission (López et al. 2011). The $1.2 \mathrm{~mm}$ emission is fragmented at a spatial resolution of $\sim 0.2 \mathrm{pc}$ and composed of $\sim 54$ clumps, which have an average mass of $75 M_{\odot}$. Nineteen clumps are associated with infrared sources identified in the Midcourse Space Experiment (MSX; Price et al. 2001) and Spitzer observations (Benjamin et al. 2003), including the source IRAS 16562-3959, a massive star-forming region (MSFR) with a luminosity of $\sim 5.3 \times 10^{4} L_{\odot}$ and associated with the most massive and dense clump in the ring. Within the ring lie also 35 cold clumps, that is, dense condensations that are not associated with an infrared counterpart. They might be regarded as candidates in which massive star formation has not yet started.

\section{Observations}

\section{1. ${ }^{13} \mathrm{CO}(3-2)$ line}

The ${ }^{13} \mathrm{CO}(3-2)$ line observations were made at $330.588 \mathrm{GHz}$ using on-the-fly (OTF) observing mode with the APEX-2A heterodyne receiver mounted at the APEX telescope (Güsten et al. 2006; Risacher et al. 2006). The whole ring-like structure G345.45+1.50 was mapped using a total time on source of $\sim 14$ h on October 17-19, 2005, May 28, June 24-26, and October $7-10,2006$. The steps in the map were $6 \operatorname{arcsec}$ and the dump integration time was $1.0 \mathrm{~s}$. At $331 \mathrm{GHz}$ the telescope has a beam size of $18^{\prime \prime}$, corresponding to $\sim 0.2 \mathrm{pc}$ at $1.8 \mathrm{kpc}$ from the Sun, and a main-beam efficiency of $\sim 0.73$. The spectrometer had a bandwidth of $1 \mathrm{GHz}$ with 8192 channels, giving a spectral resolution of $122 \mathrm{kHz}$, corresponding to $\sim 0.1 \mathrm{~km} \mathrm{~s}^{-1}$ at this frequency.

The observations were reduced using $\mathrm{XS}^{2}$ software, resulting in a data cube with a projected size of $\sim 17 \times 22 \operatorname{arcmin}^{2}$ and a pixel size of $20^{\prime \prime}$, consisting of 3036 spectra with an average rms of $\sim 1 \mathrm{~K}$ (antenna temperature) at a spectral resolution of $\sim 0.1 \mathrm{~km} \mathrm{~s}^{-1}$.

\section{2. ${ }^{12} \mathrm{CO}(3-2)$ line}

To estimate the gas temperature in the region, the ${ }^{12} \mathrm{CO}(3-2)$ line was observed using ASTE toward 29 intensity peaks found in the ${ }^{13} \mathrm{CO}(3-2)$ line map. Observations were made on $\mathrm{Au}$ gust 272010 using the position-switching observing mode. Table 1 lists the positions of the observed peaks. The CATS$345 \mathrm{GHz}$ receiver was used, with on-source integration times of between 20 and $30 \mathrm{~s}$. The bandwidth of the spectrometer was $128 \mathrm{MHz}$ with 1024 channels, providing a spectral resolution of $125 \mathrm{kHz}$, or $\sim 0.1 \mathrm{~km} \mathrm{~s}^{-1}$ at the ${ }^{12} \mathrm{CO}(3-2)$ line transition frequency, $\sim 345.796 \mathrm{GHz}$. At this frequency, the beam size of the ASTE telescope is $\sim 22^{\prime \prime}, \sim 0.2 \mathrm{pc}$ at $1.8 \mathrm{kpc}$ from the Sun. The estimated main-beam efficiency of the telescope is $\sim 0.7$.

\footnotetext{
1 Downloadable from http://irsa.ipac. caltech.edu

2 http://www.chalmers.se/rss/oso-en/observations/ data-reduction-software
}

Table 1. Observations with the ASTE telescope of the ${ }^{12} \mathrm{CO}(3-2)$ line.

\begin{tabular}{cccc}
\hline \hline RA & Dec & ${ }^{1} T_{\mathrm{A}}^{*}$ & ${ }^{1} T_{\mathrm{K}}$ \\
$\mathrm{J} 2000$ & $\mathrm{~K}$ & $\mathrm{~K}$ \\
\hline $16: 59: 08$ & $-40: 13: 57$ & 34 & 57 \\
$16: 59: 41$ & $-40: 03: 37$ & 32 & 53 \\
$16: 59: 44$ & $-40: 10: 17$ & 23 & 40 \\
$16: 59: 46$ & $-40: 11: 37$ & 25 & 43 \\
$16: 59: 20$ & $-40: 11: 17$ & 20 & 36 \\
$16: 59: 13$ & $-40: 11: 17$ & 23 & 41 \\
$16: 59: 08$ & $-40: 05: 37$ & 19 & 35 \\
$16: 59: 32$ & $-40: 10: 17$ & 15 & 29 \\
$16: 59: 35$ & $-40: 07: 37$ & 26 & 45 \\
$16: 59: 04$ & $-40: 11: 17$ & 20 & 36 \\
$16: 59: 23$ & $-40: 13: 17$ & 16 & 30 \\
$16: 59: 35$ & $-40: 12: 57$ & 21 & 38 \\
$16: 59: 53$ & $-40: 08: 37$ & 9.7 & 21 \\
$16: 59: 20$ & $-40: 09: 37$ & 14 & 28 \\
$16: 59: 11$ & $-40: 02: 37$ & 14 & 27 \\
$16: 59: 28$ & $-39: 57: 57$ & 13 & 25 \\
$16: 59: 23$ & $-39: 58: 37$ & 14 & 27 \\
$16: 59: 51$ & $-40: 00: 37$ & 18 & 34 \\
$16: 59: 44$ & $-39: 59: 17$ & 12 & 25 \\
$16: 59: 32$ & $-39: 58: 17$ & 13 & 26 \\
$16: 59: 16$ & $-40: 00: 57$ & 15 & 29 \\
$16: 59: 18$ & $-39: 58: 57$ & 13 & 26 \\
$16: 59: 30$ & $-39: 56: 37$ & 9.6 & 21 \\
$16: 59: 06$ & $-39: 55: 57$ & 11 & 23 \\
$16: 58: 50$ & $-40: 08: 16$ & 13 & 26 \\
$16: 59: 56$ & $-39: 58: 37$ & 11 & 23 \\
$16: 59: 49$ & $-39: 56: 17$ & 7.2 & 17 \\
$16: 58: 52$ & $-40: 09: 16$ & 15 & 29 \\
$16: 59: 58$ & $-40: 05: 57$ & 7.7 & 18 \\
\hline & & & \\
1 & & \\
16 &
\end{tabular}

Notes. Columns 1 and 2 show equatorial coordinates; Col. 3 the antenna temperature intensity peaks; and Col. 4 the estimated kinetic temperatures. ${ }^{(1)}$ The error for $T_{\mathrm{A}}^{*}$ and $T_{\mathrm{K}}$ values is $\sim 0.2 \mathrm{~K}$.

The data were reduced using the NEWSTAR ${ }^{3}$ software, resulting in 29 spectra with an antenna temperature rms of $\sim 0.2 \mathrm{~K}$ at a spectral resolution of $0.1 \mathrm{~km} \mathrm{~s}^{-1}$.

\section{Results and discussion}

\section{1. ${ }^{13} \mathrm{CO}(3-2)$ line}

The ${ }^{13} \mathrm{CO}(3-2)$ line toward the ring has velocities between -30 and $-2 \mathrm{~km} \mathrm{~s}^{-1}$, with two gas components; the stronger peaks at $-12.4 \mathrm{~km} \mathrm{~s}^{-1}$ and the weaker at $-25 \mathrm{~km} \mathrm{~s}^{-1}$. Figure 1 shows the globally averaged spectrum of the ${ }^{13} \mathrm{CO}(3-2)$ line over the whole ring. The velocity range agrees with the hypothesis that the ring is part of GMC G345.5+1.0, which has velocity components of between -33 and $-2 \mathrm{~km} \mathrm{~s}^{-1}$. The two velocity components observed in the ${ }^{13} \mathrm{CO}(3-2)$ line were previously identified in the ${ }^{12} \mathrm{CO}(1-0)$ line (Bronfman et al. 1989), and the stronger component in the CS(2-1) line (Bronfman et al. 1996).

\footnotetext{
3 www.nro.nao.ac.jp/\$ sim\$nro45mrt/obs/newstar
} 


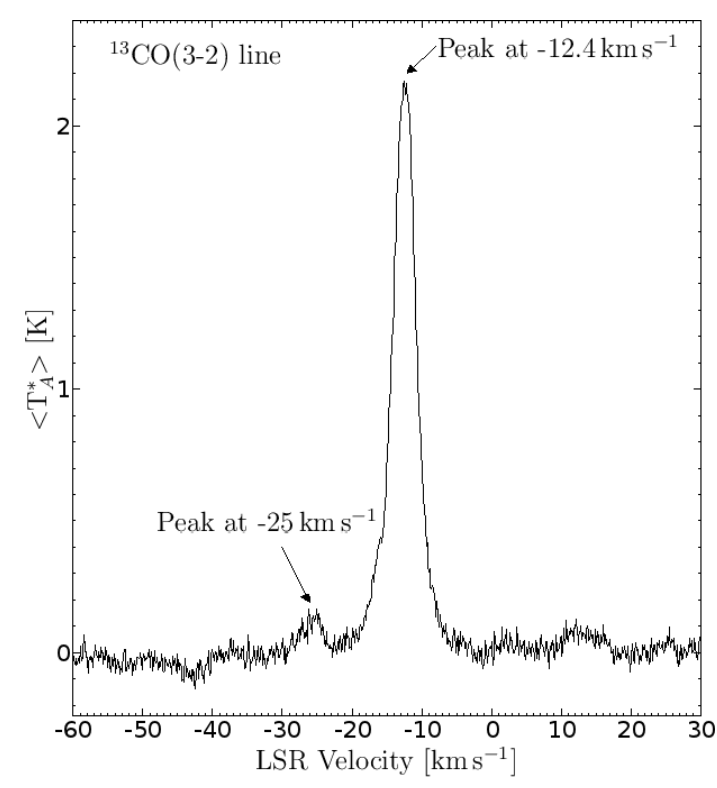

Fig. 1. Average of all ${ }^{13} \mathrm{CO}(3-2)$ spectra observed toward the ring-like structure G345.45+1.50. $\left\langle T_{\mathrm{A}}^{*}\right\rangle$ denotes the antenna temperature average. Arrows indicate the two peaks in the emission.

The spatial distributions of the ${ }^{13} \mathrm{CO}(3-2)$ line for the whole ring and for the two velocity components are shown in Figs. 2a-c, integrated in three velocity ranges, -30 to $-2 \mathrm{~km} \mathrm{~s}^{-1},-26$ to $-24 \mathrm{~km} \mathrm{~s}^{-1}$, and -22 to $-2 \mathrm{~km} \mathrm{~s}^{-1}$, respectively. To display the weaker component, the velocity range -26 to $-24 \mathrm{~km} \mathrm{~s}^{-1}$ was used because the resulting integrated image is less noisy than that using the velocity range -30 to $-22 \mathrm{~km} \mathrm{~s}^{-1}$. The spatial structure of the stronger component $\left(-22\right.$ to $\left.-2 \mathrm{~km} \mathrm{~s}^{-1}\right)$ contains most of the emission and is composed of several condensations that form a ring-like shape. Figure $2 \mathrm{c}$ shows that the weaker component corresponds to a small condensation.

\subsection{Star formation process along the ring}

It is possible to distinguish different stages in the star formation process along the ring by comparing the ${ }^{13} \mathrm{CO}(3-2)$ line with observations made at other wavelengths.

Figure 3 shows the $1.2 \mathrm{~mm}$ continuum emission overlaid with contours of the integrated ${ }^{13} \mathrm{CO}$ line intensity. The ${ }^{13} \mathrm{CO}(3-2)$ line delineates the cold dust structure traced in $1.2 \mathrm{~mm}$ continuum emission, exhibiting the same ring-like structure; but the ${ }^{13} \mathrm{CO}(3-2)$ line is more extended than the $1.2 \mathrm{~mm}$ continuum. These cold gas and dust components enclose the emission generated by hot dust heated by embedded stars, which are observed in the MSX and Spitzer images displayed in Figs. $4 \mathrm{a}-\mathrm{c}$.

Cold condensations are the sites where stars might eventually be formed. These condensations are identified along the ring, being traced by the ${ }^{13} \mathrm{CO}(3-2)$ line or the $1.2 \mathrm{~mm}$ continuum, but without an infrared counterpart in MSX and Spitzer images, mainly in $21.34 \mu \mathrm{m}$ MSX band, since the other infrared bands are contaminated by polycyclic aromatic hydrocarbon (PAH) emission and photospheric emission from stars (e.g. Chavarría et al. 2008).

Figure $4 \mathrm{~d}$ shows the $35.6 \mathrm{~cm}$ continuum image taken from Molonglo Galactic Plane Survey (MGPS; Murphy et al. 2007), which is thought to correspond mainly to free-free emission, tracing HII regions. These HII regions have counterparts at infrared wavelengths; J165920-400424, a $35.5 \mathrm{~cm}$ source from MGPS located at RA $=16: 59: 29$ Dec $=-40: 04: 23$ (J2000), does not have a clear infrared counterpart, however. It might be a pulsar produced by a supernova explosion (see Sect. 3.11), but other possibilities such as an extragalactic source, a radio star, or planetary nebulae cannot be ruled out (e.g. Whiteoak 1992). To reveal the nature of this source, the NED ${ }^{4}$ and Vizier ${ }^{5}$ databases were used. No object was found within 2 arcmin in NED, and only stellar objects are found in Vizier within 25 arcsec, approximately half of the beam-size of the MGPS observations. To clarify the nature of this source, ATCA observations would be needed toward J165920-400424 in cm wavelengths, allowing us to determine whether the emission is synchrotron or bremsstrahlung.

Features at 7.7 and $8.6 \mu \mathrm{m}$, attributed to vibrational emission of PAHs excited by UV radiation (e.g. van Dishoeck 2004), should be observed in the $8.0 \mu \mathrm{m}$ Spitzer band, while the $4.5 \mu \mathrm{m}$ Spitzer band is expected to contain no PAH features, and therefore traces the continuum emission (Benjamin et al. 2003). The emission in the 4.5 and $8.0 \mu \mathrm{m}$ bands toward the ring are roughly similar, but the $8.0 \mu \mathrm{m}$ emission is more extended and delineates the structures better, possible by emission of PAHs.

\subsection{Gas temperature}

The gas kinetic temperature, $T_{\mathrm{k}}$, was estimated from our ${ }^{12} \mathrm{CO}(3-2)$ observations by assuming optically thick emission and that the excitation temperature can be approximated by the kinetic temperature. The peak brightness, $T_{\mathrm{B}}$, of the line is then

$T_{\mathrm{B}}=\frac{h v}{k} \frac{1}{\exp \left(h v / k T_{\mathrm{k}}\right)-1}$,

where $v$ is the frequency, $h$ and $k$ are the Planck and Boltzmann constants, respectively. We recall that estimating $T_{\mathrm{k}}$ in this way results in a lower limit, since the source may not fill the beam entirely. Absorption taking place in lower excitation foreground gas will also yield a lower estimate. For example, Fig. 2d shows a spectrum without absorption, but Figs. $2 \mathrm{e}$ and $\mathrm{f}$ show signs of self-absorption in some positions. Assuming a beam-filling factor $\left(f_{\mathrm{BEAM}}\right)$ of $1, T_{\mathrm{B}}$ can be determined from

$f_{\mathrm{BEAM}} T_{\mathrm{B}} \sim T_{\mathrm{MB}} \sim T_{\mathrm{A}}^{*} / \eta_{\mathrm{MB}}$,

where $T_{\mathrm{MB}}$ is the peak of the main-beam brightness temperature, $T_{\mathrm{A}}^{*}$ is the peak of the antenna temperature, and $\eta_{\mathrm{MB}}$ is the mainbeam efficiency, $\sim 0.7$.

$T_{\mathrm{A}}^{*}$ varies between 7.2 and $34 \mathrm{~K}$, implying a variation of $T_{\mathrm{K}}$ between 17 and $57 \mathrm{~K}$, with an average value of $\sim 30 \mathrm{~K}$ as is shown in Table 1, consistent with the dust temperature of $\sim 30 \mathrm{~K}$ estimated for MSFRs (Faúndez et al. 2004).

\subsection{Column density of the ring}

Assuming that the ${ }^{13} \mathrm{CO}(3-2)$ line emission is optically thin and in local thermal equilibrium (LTE), the total column density can be determined using Eq. (A.7). From the integrated ${ }^{13} \mathrm{CO}(3-2)$ line intensity between -30 and $-2 \mathrm{~km} \mathrm{~s}^{-1}$, the column density of the ring varies between $2 \times 10^{21}$ and $7 \times 10^{22} \mathrm{~cm}^{-2}$, with an average of $7 \times 10^{21} \mathrm{~cm}^{-2}$.

The column density of the ring can also be estimated from the $1.2 \mathrm{~mm}$ dust continuum emission, $N_{1.2} \mathrm{~mm}$. This emission is

\footnotetext{
4 http://ned.ipac.caltech.edu/

5 http://vizier.u-strasbg.fr/viz-bin/VizieR
} 

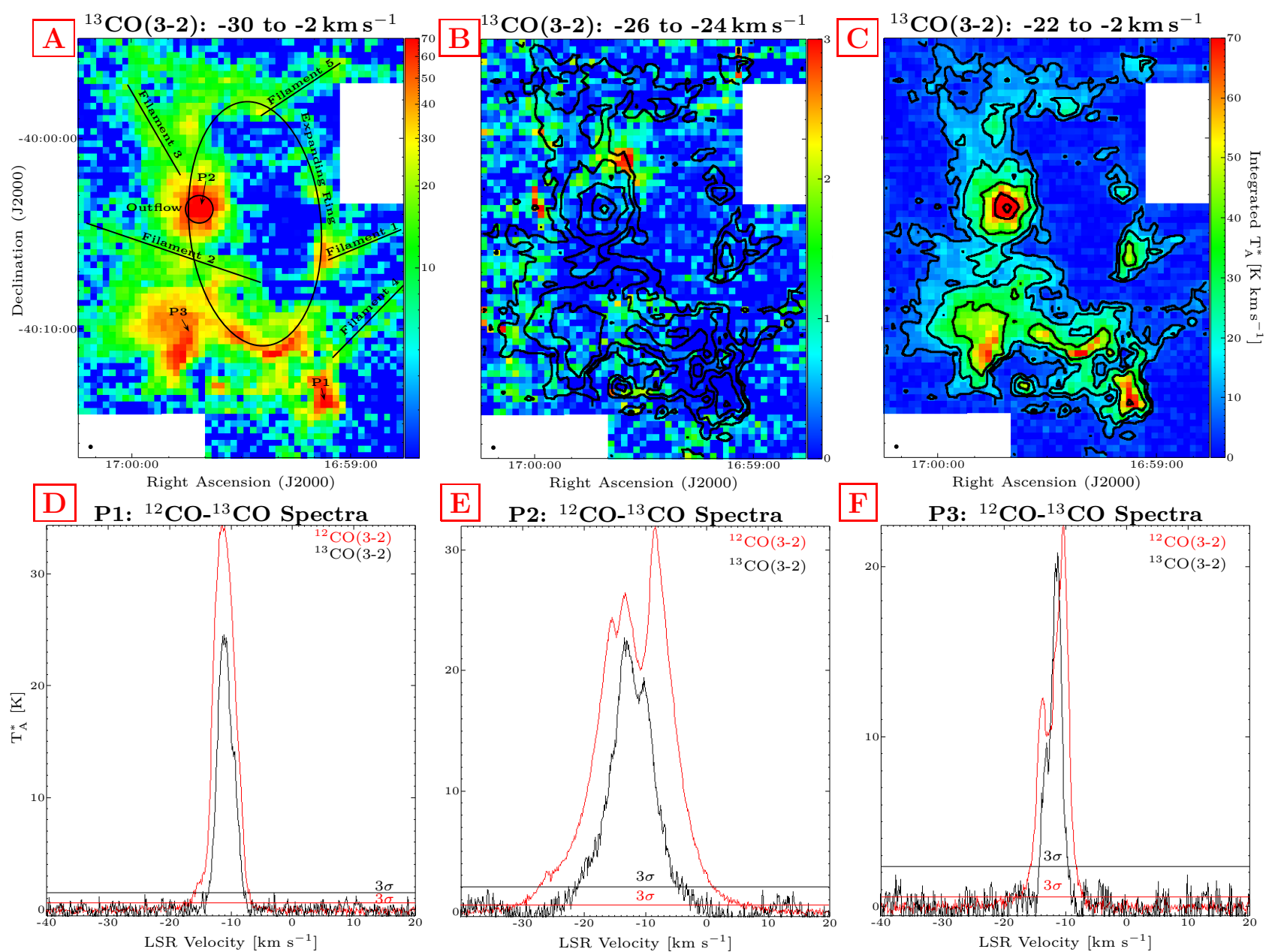

Fig. 2. Top panels: integrated ${ }^{13} \mathrm{CO}(3-2)$ line of the ring $\mathrm{G} 345.50+1.50$ between -30 and $-2 \mathrm{~km} \mathrm{~s}^{-1}$ (panel A)), between -26 and $-24 \mathrm{~km} \mathrm{~s}{ }^{-1}$ (panel B)), and between -22 and $-2 \mathrm{~km} \mathrm{~s}^{-1}$ (panel C)). The black circles in the bottom left corner represent the APEX beamsize at $330.588 \mathrm{GHz}$, $\sim 18^{\prime \prime}$. Contours show the emission integrated between -30 and $-2 \mathrm{~km} \mathrm{~s}^{-1}$ with levels of 9, 18, 36, 72, and $144 \mathrm{~K} \mathrm{~km} \mathrm{~s}^{-1}$. In panel A), the ellipse indicates the expanding ring model in the $X-Y$ plane, the lines show the filaments, and the circle plots the outflow IRAS 16562-3959. Panels D)-F) show the ${ }^{12} \mathrm{CO}(3-2)$ and ${ }^{13} \mathrm{CO}(3-2)$ spectra toward the positions $\mathrm{P} 1, \mathrm{P} 2$, and $\mathrm{P} 3$, respectively, indicated in panel A) with the arrows.

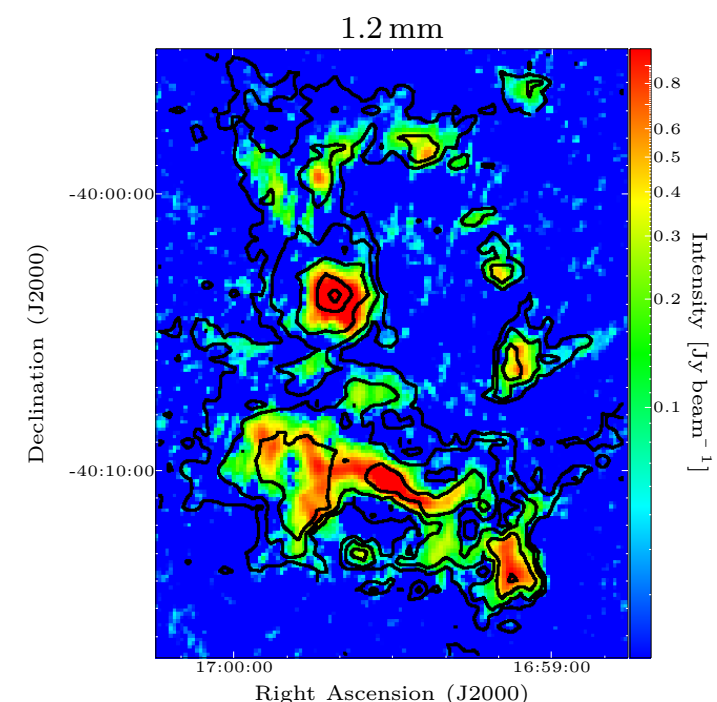

Fig. 3. $1.2 \mathrm{~mm}$ continuum emission of the ring $\mathrm{G} 345.50+1.50$ (López et al. 2011), with contours of the ${ }^{13} \mathrm{CO}(3-2)$ line integrated between -30 and $-2 \mathrm{~km} \mathrm{~s}^{-1}$ (levels: 9, 18, 36, 72, and $144 \mathrm{~K} \mathrm{~km} \mathrm{~s}^{-1}$ ).

assumed to be optically thin, thus the total column density is given by Eq. (B.1).
Figure 5 shows the ratio of $N_{1.2 \mathrm{~mm}}$ to $N_{{ }^{13} \mathrm{CO}}$ overlaid with contours of the integrated ${ }^{13} \mathrm{CO}(3-2)$ line, which is proportional to the column density following Eq. (B.1). The ratio $N_{1.2 \mathrm{~mm}} / N_{13} \mathrm{CO}$ varies from $\sim 0.1$ to 10 with an average value of $\sim 1.0 . N_{1.2 \mathrm{~mm}}$ is higher than $N_{{ }^{13} \mathrm{CO}}$ toward the central and dense parts of the condensations. This is likely caused by optically thick ${ }^{13} \mathrm{CO}$ emission. To investigate this in more detail, we would need $\mathrm{C}^{18} \mathrm{O}$ observations toward the central parts and also continuum data at shorter wavelengths than $1.2 \mathrm{~mm}$. Low $N_{1.2 \mathrm{~mm}} / N_{{ }^{3} \mathrm{CO}}$ ratios are generally found near the edges of the condensations where the density is presumingly lower. The reason for this is not clear, but $T_{\mathrm{K}}>T_{\text {dust }}$ or different dust properties near the edges might explain it.

The assumed values of the physical parameters can introduce variations in the estimated ratio $N_{1.2 \mathrm{~mm}} / N_{{ }^{13} \mathrm{CO}}$. For example, Faúndez et al. (2004) found massive star-forming regions with dust temperatures of between 18 and $46 \mathrm{~K}$, which produce variations in this ratio by a factor 2 . Hildebrand (1983) estimated a ratio of gas to dust masses of 100 with errors within a factor 2-8. Frerking et al. (1982) estimated a $\left[\mathrm{H}_{2} /{ }^{13} \mathrm{CO}\right]$ of $7 \times 10^{5}$ with possible variations of a factor 2-10. Ossenkopf \& Henning (1994) computed a dust opacity of $\sim 1 \mathrm{~cm}^{2} / \mathrm{g}$ at $1.2 \mathrm{~mm}$, with deviations no more than a factor 2 . Beam dilution is another source of error; when there is beam dilution, the ratio $N_{1.2 \mathrm{~mm}} / N_{{ }^{3} \mathrm{CO}}$ has to 

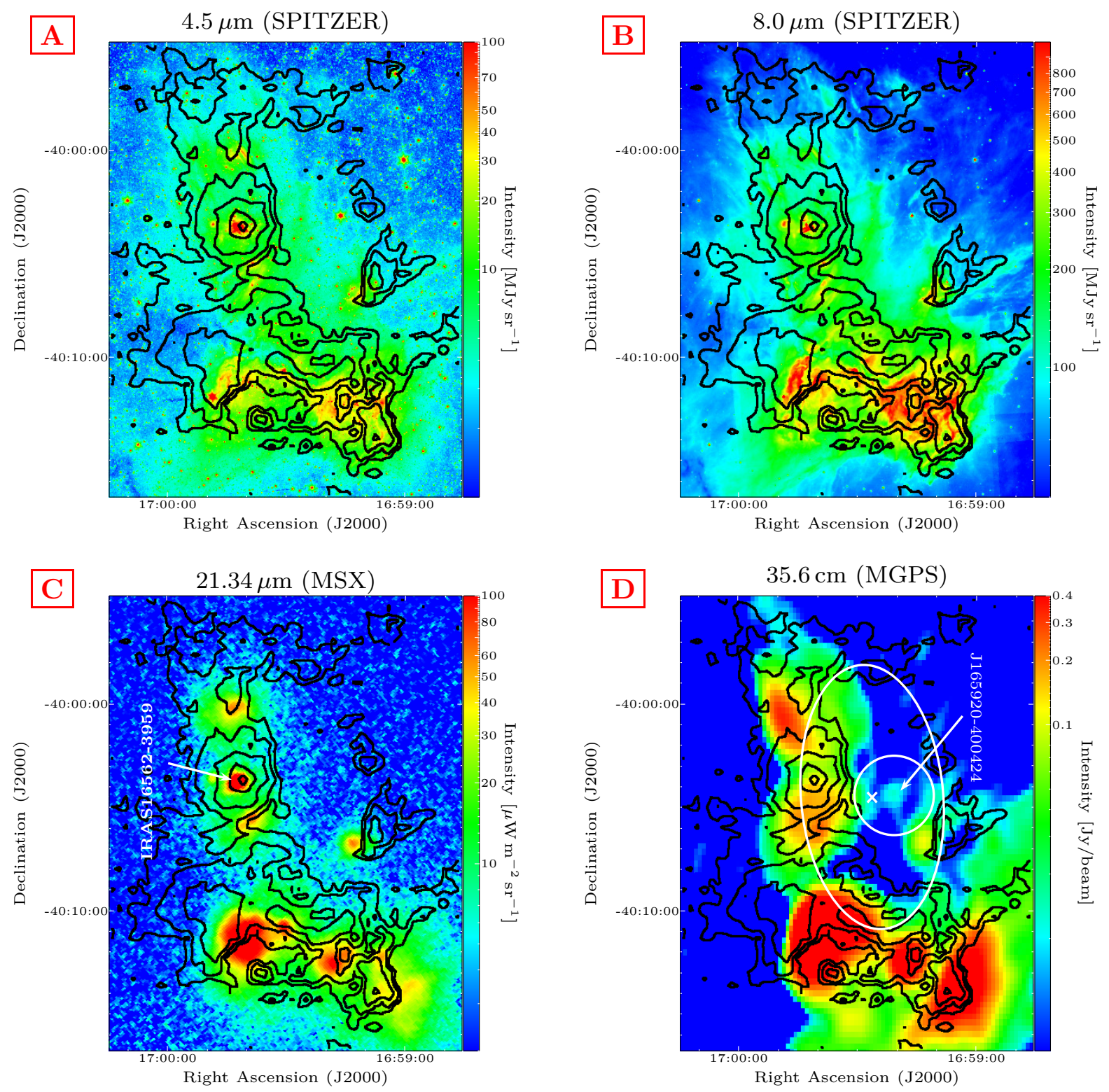

Fig. 4. Images of radio and infrared continuum emission toward the ring $\mathrm{G} 345.50+1.50$, overlaid with contours of the ${ }^{13} \mathrm{CO}(3-2)$ line integrated between -30 and $-2 \mathrm{~km} \mathrm{~s}^{-1}$ (levels: $9,18,36,72$, and $144 \mathrm{~K} \mathrm{~km} \mathrm{~s}^{-1}$ ). Panels A) and B): 4.5 and $8.0 \mu \mathrm{m}$ images from Spitzer. Panel C): $21.34 \mu \mathrm{m}$ image from MSX. Panel D): $35.6 \mathrm{~cm}$ image from MGPS (Murphy et al. 2007). The arrow indicates the position of the $35.6 \mathrm{~cm}$ source J165920400424 , and the $2^{\prime}$ white circle shows the area where objects were searched for in the NED database to determine the nature of this source. The ellipse indicates the expanding ring model in the $X-Y$ plane, with its center indicated with an $X$, see Sect. 3.11.

be corrected by a factor $\Omega_{\mathrm{MB}-\mathrm{SEST}}^{2} / \Omega_{\mathrm{MB}-\mathrm{APEX}}^{2} \sim\left(24^{\prime \prime}\right)^{2} /\left(18^{\prime \prime}\right)^{2} \sim$ 1.8 , where $\Omega_{\text {MB-SEST }}$ is the main-beam solid angle for SEST, and $\Omega_{\text {MB-APEX }}$, for APEX. CO freeze-out on dust grains may also affect the $N_{1.2} \mathrm{~mm} / N_{{ }^{13} \mathrm{CO}}$ ratio in regions with low temperatures $(<15 \mathrm{~K})$ and high densities $\left(>10^{4} \mathrm{~cm}^{-3}\right.$; Kramer et al. 1999).

\subsection{Mass of the ring}

Following Eqs. (A.8) and (B.2), it is possible to obtain two different measurements of the total mass of the ring using the ${ }^{13} \mathrm{CO}(3-2)$ line and the $1.2 \mathrm{~mm}$ continuum emission independently. The total integrated emission in the ${ }^{13} \mathrm{CO}(3-2)$ line is $\sim 1.8 \times 10^{7} \mathrm{~K} \mathrm{~km} \mathrm{~s}^{-1} \operatorname{arcsec}^{2}$, implying that the derived total mass is $6.9 \times 10^{3} M_{\odot}$. The total flux density in the $1.2 \mathrm{~mm}$ continuum emission is $\sim 120 \mathrm{Jy}$, giving a total mass of $\sim 4.0 \times 10^{3} \mathrm{M}_{\odot}$. Considering the errors of the assumed physical parameters (see Sect. 3.4), the mass estimates agree well.

\subsection{Identification of the ${ }^{13} \mathrm{CO}$ clumps}

As Figs. $2 b$ and $c$ show, the ${ }^{13} \mathrm{CO}(3-2)$ line emission is clumpy. Using CLUMPFIND ${ }^{6}$ (Williams et al. 1994), 104 clumps $\left({ }^{13} \mathrm{CO}\right.$ clumps) were identified. They contain $65 \%$ of the total integrated emission, $\sim 1.2 \times 10^{7} \mathrm{~K} \mathrm{~km} \mathrm{~s}^{-1} \operatorname{arcsec}^{2}$. CLUMPFIND creates

6 http://www.ifa.hawai i.edu/users/jpw/clumpfind.shtml 


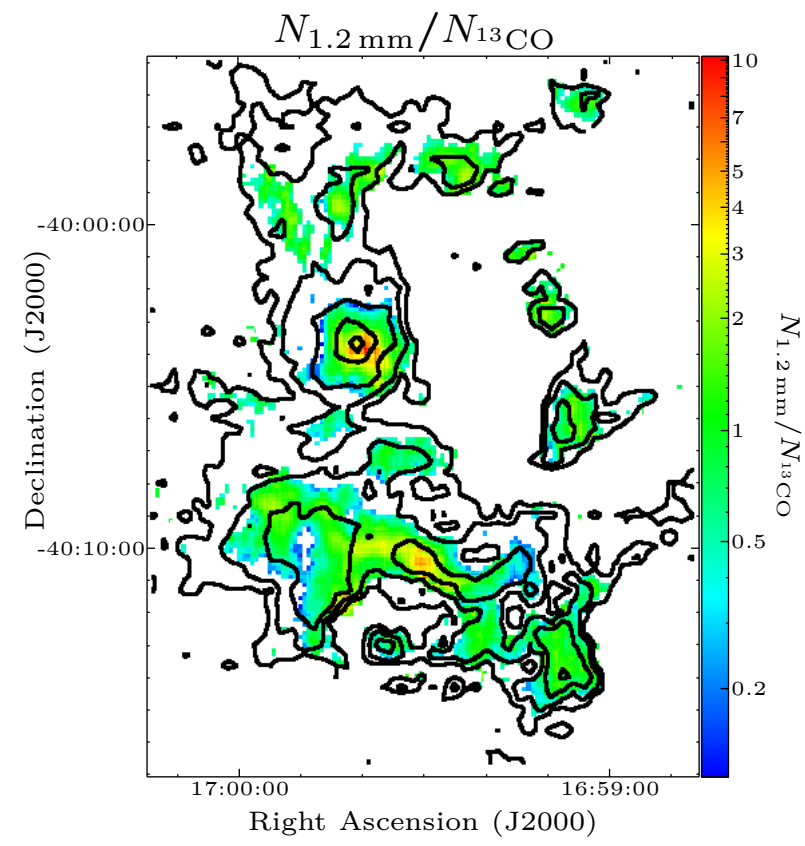

Fig. 5. Map of the column density ratio $N_{1.2 \mathrm{~mm}} / N_{13} \mathrm{CO}$ with contours of the ${ }^{13} \mathrm{CO}(3-2)$ line integrated between -30 and $-2 \mathrm{~km} \mathrm{~s}^{-1}$ (levels: 9 , $18,36,72$, and $144 \mathrm{~K} \mathrm{~km} \mathrm{~s}^{-1}$ ). Only significant column density values, higher than $3 \sigma$, have been used in the ratio map.

contours over the data, searches for peaks of emission to locate clumps, and follows them down to the lower intensity contour. To find these clumps, the algorithm was applied with a lower intensity contour of three rms and with a contouring interval equal to twice the rms. Fictitious clumps were filtered out by the condition that intensity peaks have to be higher than five times the rms, $\sim 5 \mathrm{~K}$.

\subsection{Evolution of clumps}

The evolution of the clumps can be investigated by associating the ${ }^{13} \mathrm{CO}$ clumps with the sources detected in $1.2 \mathrm{~mm}$ continuum and MSX observations.

The clumps are classified into three types, A, B, and C. Type A clumps have a counterpart seen in infrared and millimeter wavelengths, type B clumps only have a counterpart in the $1.2 \mathrm{~mm}$ continuum, and type $\mathrm{C}$ clumps are only seen in ${ }^{13} \mathrm{CO}$.

The criterion for correlating the sources was to identify the centers closer than $\sim 27^{\prime \prime}, 1.5$ beam sizes of the ${ }^{13} \mathrm{CO}$ line observations. Since the $8.3 \mu \mathrm{m}$ MSX band is sensitive to the PAH emission and to the photospheric emission from stars (e.g. Chavarría et al. 2008), clumps not detected in all MSX bands are considered to have no counterpart at infrared wavelengths.

About $6 \%$ of the ${ }^{13} \mathrm{CO}$ clumps are type A, $27 \%$ of the clumps are type $\mathrm{B}$, and the remaining clumps, $67 \%$, are type $\mathrm{C}$.

Owing to the sensitivity limit of the MSX observations, the percentage of type A clumps is a lower limit to the number of clumps that are forming stars, and the percentages of types B and $\mathrm{C}$ clumps are an upper limit to the number of clumps that are not forming stars.

As the ${ }^{13} \mathrm{CO}(3-2)$ line is more sensitive to lower densities than the $1.2 \mathrm{~mm}$ continuum (see Sect. 3.4 ), type $\mathrm{C}$ clumps have to correspond to structures with low densities that can represent early states of the clumps.

Assuming that type A clumps are embedded clusters with typical ages of $2.5 \mathrm{Myr}$ (Lada \& Lada 2003) and that the timescale for each evolutionary stage is proportional to the

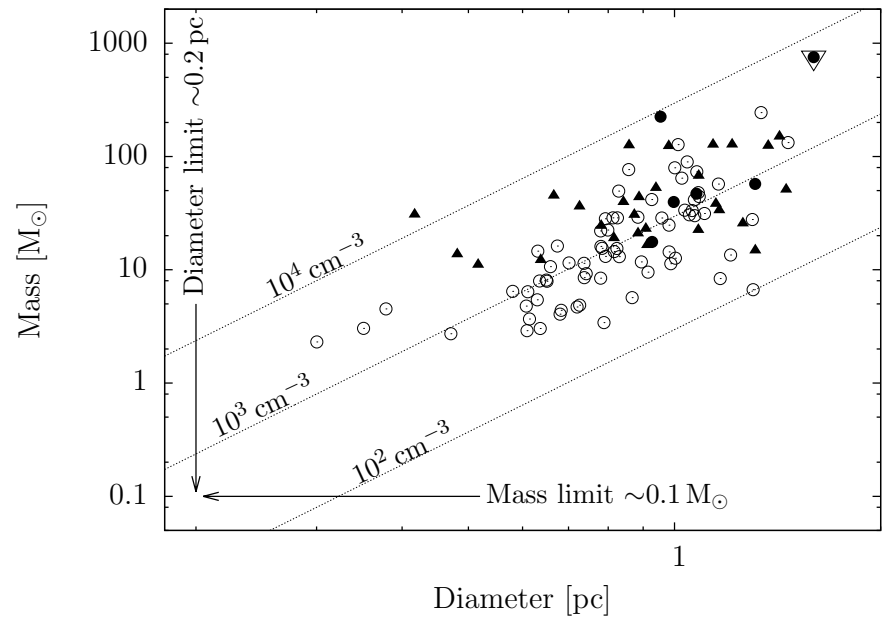

Fig. 6. Mass versus diameter for the clumps detected in the ${ }^{13} \mathrm{CO}(3-2)$ line. The filled circles represent type A clumps, the filled triangles show type B clumps, and the open circles plot the type C clumps. The open triangle indicates the clump associated with the MSFR IRAS 16562-3959. The arrows show the minimal detectable diameter and mass, $\sim 0.2 \mathrm{pc}$ and $\sim 0.1 M_{\odot}$. The dotted lines indicate mean densities at $10^{2}, 10^{3}$, and $10^{4} \mathrm{~cm}^{-3}$.

number of clumps, type B clumps have timescales of $\lesssim 11 \mathrm{Myr}$ and type $\mathrm{C}$ clumps timescales of $\lesssim 28 \mathrm{Myr}$. Thus, the typical lifetime for these clumps probably is $\lesssim 40 \mathrm{Myr}$, an upper limit comparable with the GMC lifetimes of 20-40 Myr (Blitz 1993; Kawamura et al. 2009; Miura et al. 2012).

\subsection{Physical properties of the ${ }^{13} \mathrm{CO}$ clumps}

Using Eq. (A.8), the derived masses of the clumps are between 2.3 and $7.5 \times 10^{2} M_{\odot}$, with an average value of $44 M_{\odot}$. The lowest detected clump mass is $0.1 M_{\odot}$, assuming the smallest dimension of $0.111 \mathrm{~km} \mathrm{~s}^{-1} \times 20 \operatorname{arcsec} \times 20 \operatorname{arcsec}$ and lowest antenna temperature of $5 \mathrm{~K}$ ( 5 sigma level).

Clump diameters, $D_{\mathrm{c}}$, are estimated from the deconvolved FWHM size of their emissions using Eq. (C.1). The clumps have diameters of between 0.3 and $1.6 \mathrm{pc}$ with an average of $0.9 \mathrm{pc}$.

Using the masses and diameters, the mean column density, $N_{\mathrm{c}}$, and the mean density, $n_{\mathrm{c}}$, are calculated using Eqs. (C.2) and (C.3), respectively. Clumps have column densities between $3 \times 10^{20}$ and $2 \times 10^{22} \mathrm{~cm}^{-2}$ with an average value of $3 \times 10^{21} \mathrm{~cm}^{-2}$, and densities between $10^{2}$ and $10^{4} \mathrm{~cm}^{-3}$ with an average value of $2 \times 10^{3} \mathrm{~cm}^{-3}$.

Figure 6 shows a plot of mass versus diameter for the ${ }^{13} \mathrm{CO}$ clumps, with lines to display different densities. Type A clumps are the most massive and dense in average, $\sim 1.9 \times 10^{2} M_{\odot}$ and $3.2 \times 10^{3} \mathrm{~cm}^{-3}$. One example is the clump associated with the MSFR IRAS 16562-3959, with a mass of $\sim 7.5 \times 10^{2} M_{\odot}$ and density of $6 \times 10^{3} \mathrm{~cm}^{-3}$. In the other extreme, type $\mathrm{C}$ clumps are the less massive and less dense on average, which is consistent with the comments in Sect. 3.7, where we indicated that these clumps might represent an earlier state in the clump formation.

Figure 7 shows line velocity width (FWHM), $\Delta V_{\mathrm{c}}$, versus diameter, $D_{\mathrm{c}}$, for the clumps. Line widths range between 0.4 and $4.2 \mathrm{~km} \mathrm{~s}^{-1}$ with an average value of $1.2 \mathrm{~km} \mathrm{~s}^{-1}$. The relationship between line width and diameter is given by

$\Delta V_{\mathrm{c}} \sim 1.2 \frac{\mathrm{km}}{\mathrm{s}}\left(\frac{D_{\mathrm{c}}}{\mathrm{pc}}\right)^{0.5}$ 


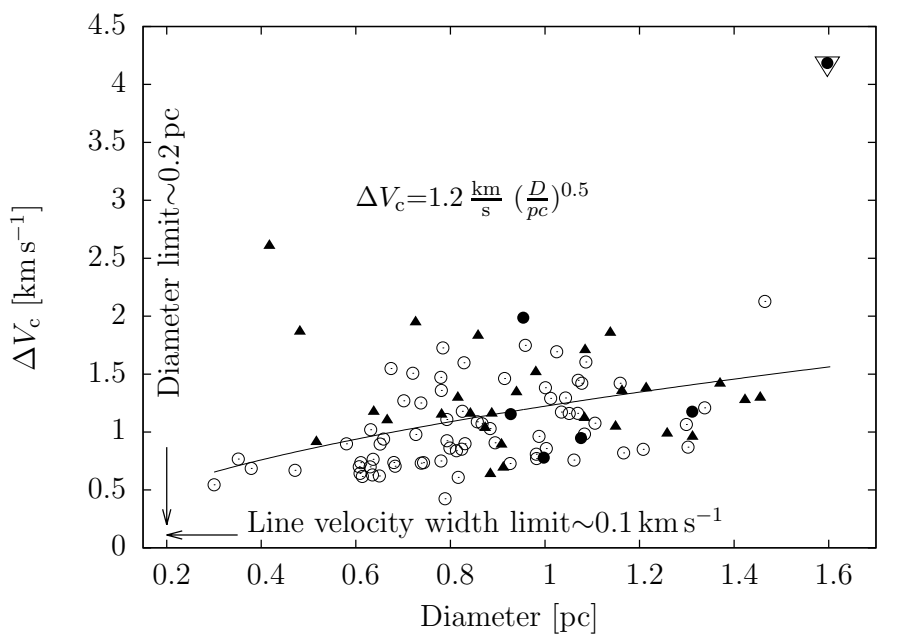

Fig. 7. Line velocity width versus diameter for the clumps detected in the ${ }^{13} \mathrm{CO}(3-2)$ line. Arrows show observational detection limits for line widths and diameters, $\sim 0.1 \mathrm{~km} \mathrm{~s}^{-1}$ and $\sim 0.2 \mathrm{pc}$. The line shows the fit, $\Delta V_{\mathrm{c}}=1.2 \mathrm{~km} \mathrm{~s}^{-1}\left(D_{\mathrm{c}} \mathrm{pc}^{-1}\right)^{0.5}$. The symbols are the same as in Fig. 6 .

This relationship is weak, with a correlation coefficient of 0.33 and dispersion of $0.5 \mathrm{~km} \mathrm{~s}^{-1}$. The clump associated with IRAS 16562-3959 shows the highest dispersion, which is explained by the outflow identified in this clump (see Sect. 3.12).

Table 2 presents a summary of the physical properties of the clumps, and Table F.1 lists the individual estimates for each clump.

The physical properties of identified clumps agree with observations toward other GMCs. For example, clumps within the GMC associated with RCW 106 detected in the ${ }^{13} \mathrm{CO}(1-0)$ line, $\mathrm{C}^{18} \mathrm{O}(1-0)$ line, and $1.2 \mathrm{~mm}$ continuum have diameters of 0.3 to $4 \mathrm{pc}$, masses of 10 to $2 \times 10^{4} M_{\odot}$, densities of $2 \times 10^{3}$ to $6 \times 10^{4} \mathrm{~cm}^{-3}$, and line velocity widths of 0.4 to $3 \mathrm{~km} \mathrm{~s}^{-1}$ (Mookerjea et al. 2004; Bains et al. 2006; Wong et al. 2008).

\subsection{Gravitational stability}

To investigate whether clumps are gravitationally bound, their physical properties were examined using two methods, assuming virial equilibrium (e.g. Bertoldi \& McKee 1992), and assuming that they can be modeled as Bonnor-Ebert spheres (Bonnor 1956).

Using the virial condition, Eq. (D.2) defines $\alpha_{\text {virial }}$, which can be used as an indicator of gravitational stability, because it is a measurement of the ratio of the kinetic energy to the gravitational energy (e.g. Wong et al. 2008). For $\alpha_{\text {virial }} \gg 1$, clumps are not bound and must be confined by an external pressure to be in hydrostatic equilibrium, for $\alpha \sim 1$, clumps are in equilibrium, and for $\alpha \ll 1$, clumps are unable to support themselves against gravity.

Figure 8 displays $\alpha_{\text {virial }}$ versus mass. All clumps have $\alpha_{\text {virial }} \gtrsim$ 1 , thus they must be in equilibrium or confined by an external pressure to be in hydrostatic equilibrium.

We employed a Bonnor-Ebert sphere analysis (see Appendix B) to determine the dimensionless radius $\xi_{\max }$ and the external pressures $P_{\text {ext }}$. When $\xi_{\max }>6.5$, the gas sphere is susceptible to gravitational collapse. In the other case, when $\xi_{\max }<6.5$, the gas sphere is thought to be stable against collapse. Figure 9 displays the dimensionless radius $\xi_{\max }$ versus mass for all clumps, and Fig. 10 shows the external pressure $P_{\text {ext }}$ versus mass for the stable clumps.

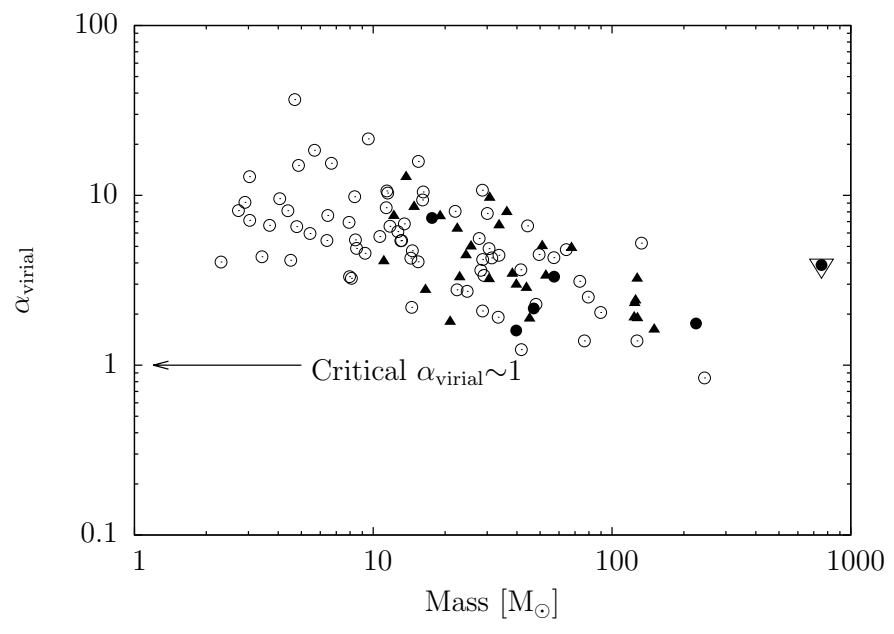

Fig. 8. $\alpha_{\text {virial }}$ versus mass for the identified clumps. The arrow indicates the critical values of $\alpha_{\text {virial }}, \sim 1$. The symbols are explained in Fig. 6 .

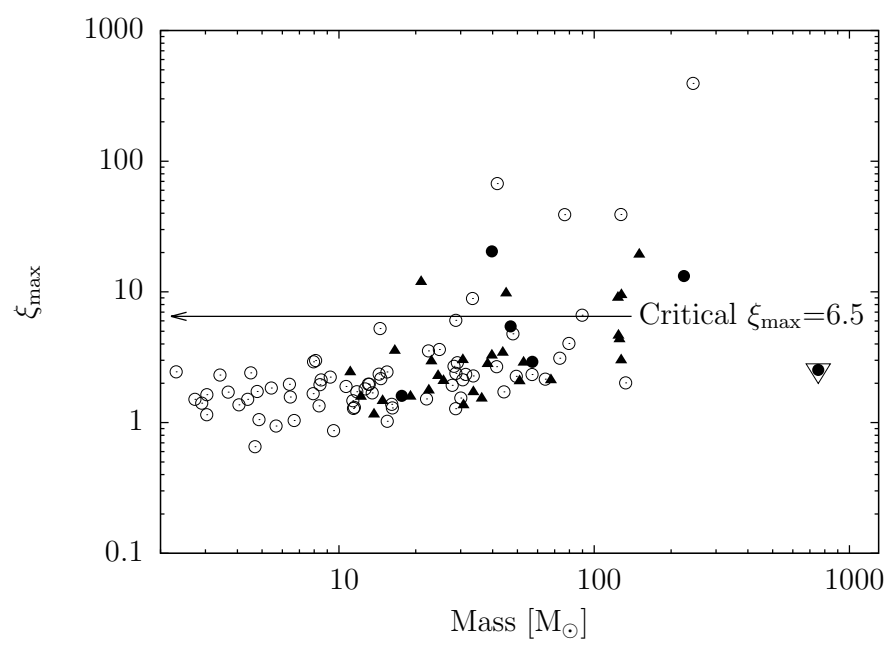

Fig. 9. Dimensionless radius $\xi_{\max }$ versus mass for the clumps detected in the ${ }^{13} \mathrm{CO}(3-2)$ line. The arrow marks the critical state for the BonnorEbert sphere $\left(\xi_{\max }=6.5\right)$. The symbols are explained in Fig. 6 .

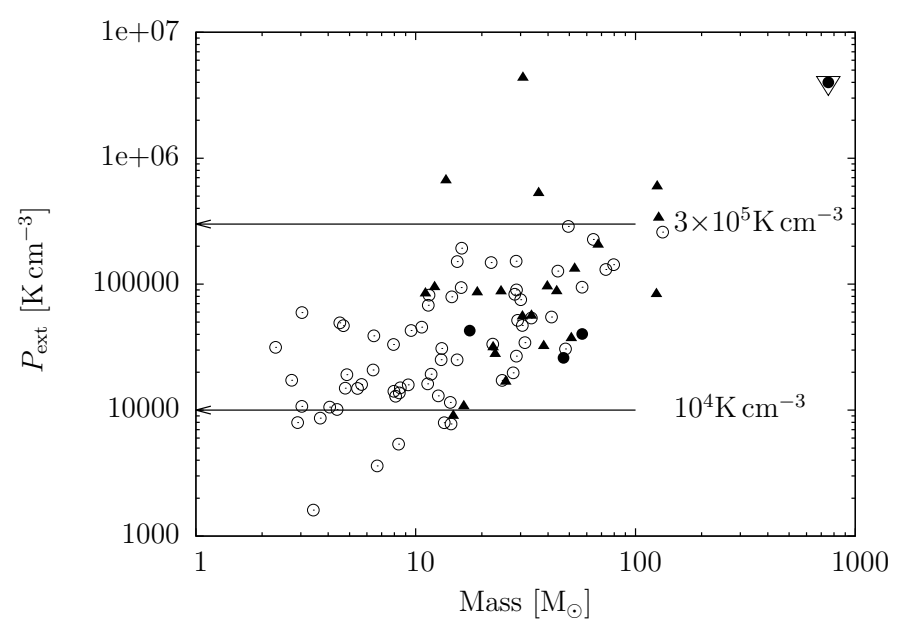

Fig. 10. External pressure $P_{\text {ext }}$ versus mass for the clumps identified in the ${ }^{13} \mathrm{CO}(3-2)$ line with $\xi_{\max }<6.5$. The arrows indicate the internal pressure estimated for the GMC G345.5+1.5 using its physical properties $\left(\sim 3 \times 10^{5} \mathrm{~K} \mathrm{~cm}^{-3}\right)$, and the mean local interstellar pressure $\left(\sim 10^{4} \mathrm{~K} \mathrm{~cm}^{-3}\right)$. The symbols are explained in Fig. 6. 
Table 2. Summary of the physical properties of the 104 identified ${ }^{13} \mathrm{CO}$ clumps.

\begin{tabular}{lccc}
\hline \hline Parameter & Range & Average & Median \\
\hline $\begin{array}{l}\text { Diameter } \\
{[\mathrm{pc}]}\end{array}$ & $0.3-1.6$ & 0.9 & 0.9 \\
${ }^{1}$ Mass & $2.3-7.5 \times 10^{2}$ & 44 & 23 \\
{$\left[M_{\odot}\right]$} & $10^{2}-10^{4}$ & $2 \times 10^{3}$ & $10^{3}$ \\
${ }^{2}$ Density & & & \\
{$\left[\mathrm{cm}^{-3}\right]$} & $3 \times 10^{20}-2 \times 10^{22}$ & $3 \times 10^{21}$ & $2 \times 10^{21}$ \\
${ }^{2} \mathrm{Column}^{-2}$ density & & & \\
{$\left[\mathrm{cm}^{-2}\right]$} & $0.7-2.0$ & 1.1 & 1.0 \\
$\begin{array}{l}\text { Line velocity width } \\
{\left[\mathrm{km} \mathrm{s}^{-1}\right]}\end{array}$ & & & \\
\hline
\end{tabular}

Notes. ${ }^{(1)}$ The total mass of the clumps is $4.5 \times 10^{3} M_{\odot} \cdot{ }^{(2)}$ Densities and column densities are estimated assuming a mean molecular weight of $\mu=2.29$.

Table 3. Summary of the physical properties of the $541.2 \mathrm{~mm}$ clumps identified in the ring G345.45+1.50 (López et al. 2011).

\begin{tabular}{lccc}
\hline \hline Parameter & Range & Average & Median \\
\hline Diameter [pc] & $0.2-0.6$ & 0.3 & 0.3 \\
Mass $\left[M_{\odot}\right]$ & $3.8-1.3 \times 10^{3}$ & 75 & 27 \\
Density $\left[\mathrm{cm}^{-3}\right]$ & $8 \times 10^{3}-3 \times 10^{5}$ & $8 \times 10^{4}$ & $5 \times 10^{4}$ \\
\hline
\end{tabular}

In general, $\xi$ seems to increase with mass, which means that with increasing mass the likelihood increases that they are in collapsing phase. However, most clumps are in hydrostatic equilibrium confined by external pressure, which partly agrees with the virial equilibrium analysis, since Bonnor-Ebert sphere analysis also shows clumps in a stage susceptible to gravitational collapse. These clumps are indicated in Table F.1.

The clumps in a hydrostatic equilibrium require external pressures of $1.6 \times 10^{3}$ to $4 \times 10^{6} \mathrm{~K} \mathrm{~cm}^{-3}$, with a median value of $\sim 4 \times 10^{4} \mathrm{~K} \mathrm{~cm}^{-3}$. This pressure represents an estimate of the pressure inside this GMC, which is consistently higher than the mean local interstellar pressure $\left(\sim 10^{4} \mathrm{~K} \mathrm{~cm}^{-3}\right.$; Bloemen 1987), but lower than the pressure required to prevent the GMC from collapsing under its own weight in virial equilibrium (e.g. Bertoldi \& McKee 1992),

$P_{\mathrm{GMC}} \sim-\frac{W_{\mathrm{GMC}}}{3 V_{\mathrm{GMC}}}=\frac{1}{5} \frac{G M_{\mathrm{GMC}}^{2}}{V_{\mathrm{GMC}} R_{\mathrm{GMC}}} \sim 3 \times 10^{5} \mathrm{~K} \mathrm{~cm}^{-3}$,

where $V_{\mathrm{GMC}}, M_{\mathrm{GMC}}$ and $R_{\mathrm{GMC}}$ are the volume $\left(\sim 4 / 3 \pi R_{\mathrm{GMC}}^{3}\right)$, mass $\left(\sim 6.5 \times 10^{5} M_{\odot}\right)$, and radius $(\sim 34 \mathrm{pc})$ of the GMC (López et al. 2011). This estimation for $P_{\mathrm{GMC}}$ is similar to the typical internal pressures of GMCs, $\sim 10^{5} \mathrm{~K} \mathrm{~cm}^{-3}$ (e.g. Blitz 1993).

\subsection{Comparison with $1.2 \mathrm{~mm}$ clumps}

A summary of the physical properties of the $541.2 \mathrm{~mm}$ clumps identified in the ring is shown in Table 3. Compared with Table 2, the typical masses of the $1.2 \mathrm{~mm}$ clumps and the ${ }^{13} \mathrm{CO}$ clumps are similar, but the $1.2 \mathrm{~mm}$ clumps are on average about three times smaller than the ${ }^{13} \mathrm{CO}$ clumps. This is consistent with the greater extension of the ${ }^{13} \mathrm{CO}(3-2)$ line emission.
The mass distributions for the $1.2 \mathrm{~mm}$ and ${ }^{13} \mathrm{CO}$ clumps are shown in Fig. 11a. From this plot, the completeness limits of the mass distributions are estimated to be $\sim 33 M_{\odot}$ and $\sim 13 M_{\odot}$ for $1.2 \mathrm{~mm}$ and ${ }^{13} \mathrm{CO}$ clumps, respectively. Bin errors are calculated as $\sqrt{\Delta N / \Delta \log \left(M / M_{\odot}\right)}$, where $\Delta N$ is the number of clumps in the constant logarithmic mass interval $\Delta \log \left(M / M_{\odot}\right), \sim 0.4$. When we consider clumps with masses higher than the completeness limits, the fits of the power law function

$$
\mathrm{d} N / \operatorname{dlog}(M) \propto M^{-\alpha+1}
$$

in the two distributions are consistent with a value for $\alpha$ of $\sim 1.9$.

As the sample of $1.2 \mathrm{~mm}$ clumps is smaller than 70 , its mass distribution can be affected by the binning (e.g. Muñoz et al. 2007). To check the value of $\alpha$ equal to 1.9 estimated from the mass distributions, the normalized cumulative mass functions of the two clump samples were calculated and are shown in Fig. 11b. To avoid the invariable increase of the slope at the end of the normalized cumulative mass function, as explained by Muñoz et al. (2007), 20\% of the total cloud mass was excluded from the fittings. The clumps with masses lower than the completeness limit were also excluded. In this range of masses, the relationship between $\alpha$ and the cumulative mass function is approximated as (Muñoz et al. 2007)

$\mathcal{N}(M) \propto M^{-\alpha+1}$

The normalized cumulative mass function and the mass distribution both show consistent values for $\alpha$ equal to $\sim 1.9$. This result also agrees with previous studies of clumps in high-mass star-forming regions made at spatial resolutions between 0.1 and 0.8 pc (e.g. Muñoz et al. 2007; López et al. 2011; Mookerjea et al. 2004; Kerton et al. 2001; Tothill et al. 2002; Kramer et al. 1998; Wong et al. 2008). Using CO isotope lines and continuum emission, these studies determined clump mass distributions with $\alpha$ of between 1.6 and 1.9. From numerical models of self-gravity molecular cloud dynamics including different levels of turbulent support, the observed range of $\alpha$ is explained by different grades in the domination of the gravity; the clump mass distribution becomes shallower when gravity increases (Klessen 2001). With these values for $\alpha$, additional fragmenting processes are needed to obtain the core mass function (e.g. Nutter \& Ward-Thompson 2007) and the star initial mass function (IMF; e.g. Kroupa 2002, 2007), which have an $\alpha$ closed to 2.35. These additional fragmenting processes are consistent with the observations, where the vast majority of stars form in binaries or higher-order multiple system (Goodwin et al. 2007).

Studies at smaller scales $(<0.1 \mathrm{pc})$ and lower masses $\left(<10 M_{\odot}\right)$ show that the core mass function has similar slopes to the stellar IMF (e.g Motte et al. 1998; Alves et al. 2007; Nutter \& Ward-Thompson 2007), arguing that the fragmentation process at this scale sets the IMF. In contrast to this hypothesis, Goodwin et al. (2008) proved that the model in which all stars and brown dwarfs form in multiple system from a core mass distribution provides a very good fit to the IMF. Our observations have a spatial resolution of $0.2 \mathrm{pc}$, therefore we cannot determine the mass function on scales $<0.1 \mathrm{pc}$.

An exception to the form of the clump mass function was found by Reid \& Wilson (2006). They rederived the clump mass function in some GMCs and found a double power-law with a slope at the high-mass end of 2.4 , but we fail to see any indication for such a distribution. 

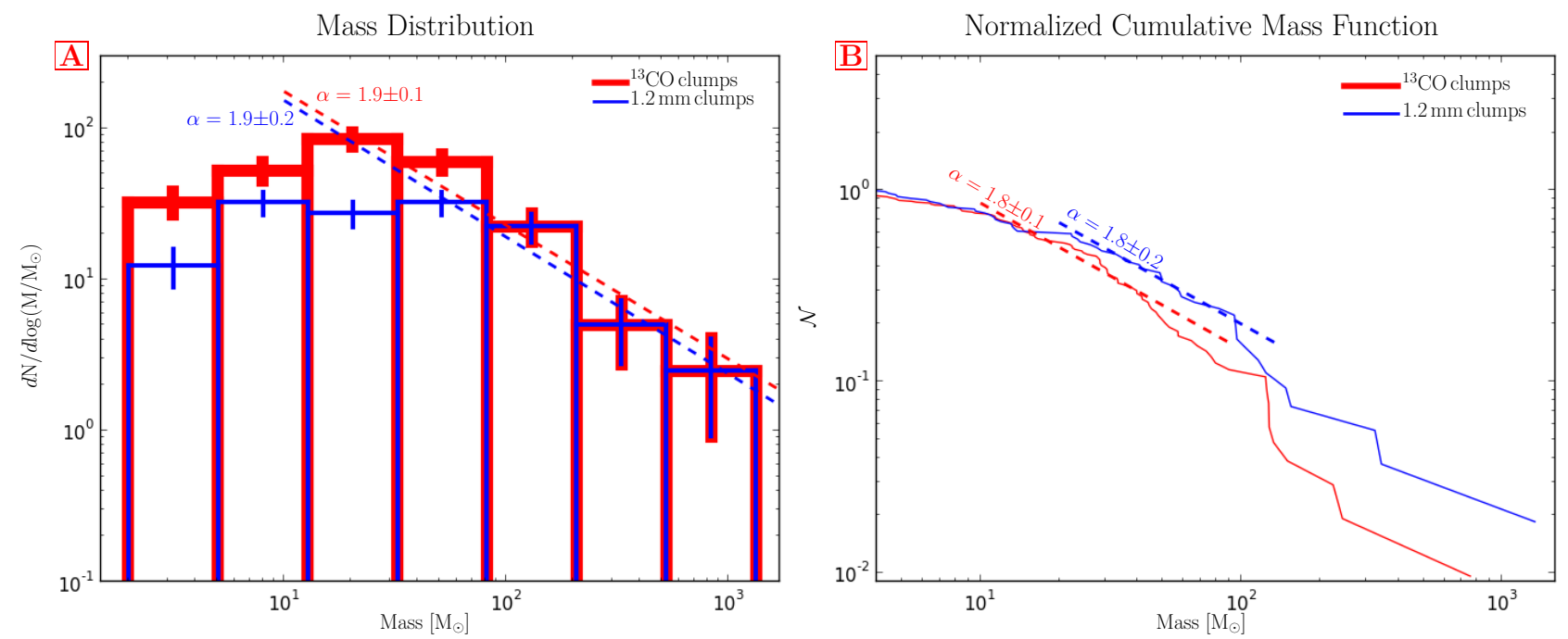

Fig. 11. Panel A) histogram of the mass distributions for the ${ }^{13} \mathrm{CO}$ clumps (solid red lines) and $1.2 \mathrm{~mm}$ clumps (solid blue lines). The dashed lines indicate the fits with $\alpha=1.9 \pm 0.1$ for ${ }^{13} \mathrm{CO}$ clumps and $\alpha=1.9 \pm 0.2$ for $1.2 \mathrm{~mm}$ clumps. Panel B) normalized cumulative mass functions for the ${ }^{13} \mathrm{CO}$ clumps (solid red lines) and $1.2 \mathrm{~mm}$ clumps (solid blue lines). The dashed lines indicate the fits with $\alpha=1.8 \pm 0.1$ for ${ }^{13} \mathrm{CO}$ clumps and $\alpha=1.8 \pm 0.2$ for $1.2 \mathrm{~mm}$ clumps.

Table 4. Characteristics of the expanding ring.

\begin{tabular}{ll}
\hline \hline Parameter & Value \\
\hline Spatial center & $16: 59: 26-40: 04: 30(\mathrm{~J} 2000)$ \\
LSR velocity center & $-12.4 \mathrm{~km} \mathrm{~s}^{-1}$ \\
Radius & $3.4 \mathrm{pc}$ \\
Expansion velocity & $1.0 \mathrm{~km} \mathrm{~s}^{-1}$ \\
$\alpha$ & $57 \mathrm{deg}$ \\
$\beta$ & $-175 \mathrm{deg}$ \\
Mass & $6.9 \times 10^{3} M_{\odot}$ \\
Energy & $7 \times 10^{46} \mathrm{erg}$ \\
Expansion time & $3 \times 10^{6} \mathrm{yr}$ \\
\hline
\end{tabular}

\subsection{Kinematic structure}

The ${ }^{13} \mathrm{CO}(3-2)$ line emission of the whole region shows the characteristics of an expanding ring-like structure, as shown in Figs. 2a and 12, where we display the velocity-integrated emission and position-velocity diagram, respectively. To characterize this structure, we used a new method: a model assuming a ring expanding at constant velocity rotated at an angle $\alpha$ in the plane $X-Z$, and rotated at an angle $\beta$ in the observed spatial plane ( $X-Y$ plane). Thus

$X(\theta)=X_{0}+R(\cos \theta \cos \alpha \cos \beta-\sin \theta \sin \beta)$

$Y(\theta)=Y_{0}+R(\cos \theta \cos \alpha \sin \beta+\sin \theta \cos \beta)$

$V_{z}(\theta)=V_{0}+V \cos \theta \sin \alpha$,

where $\theta$ is the angular position in the ring, between 0 and $2 \pi$, $X(\theta)$ and $Y(\theta)$ are the projected spatial position, $V_{z}(\theta)$ is the velocity in the $Z$ direction, $X_{0}$ and $Y_{0}$ define the spatial center, $V_{0}$ is the velocity center, $R$ is the radius, and $V$ is the expansion velocity.

The fit yields a center at RA $=16: 59: 26$, Dec $=-40: 04: 30$ $(\mathrm{J} 2000)$ and an LSR velocity $=-12.4 \mathrm{~km} \mathrm{~s}^{-1}$, with a radius and expansion velocity of $3.4 \mathrm{pc}$ and $1.0 \mathrm{~km} \mathrm{~s}^{-1}$, respectively. The expansion timescale is $\sim 3 \times 10^{6} \mathrm{yr}(t=R / V)$, and considering a total mass of $6.9 \times 10^{3} M_{\odot}$, the total kinematic energy

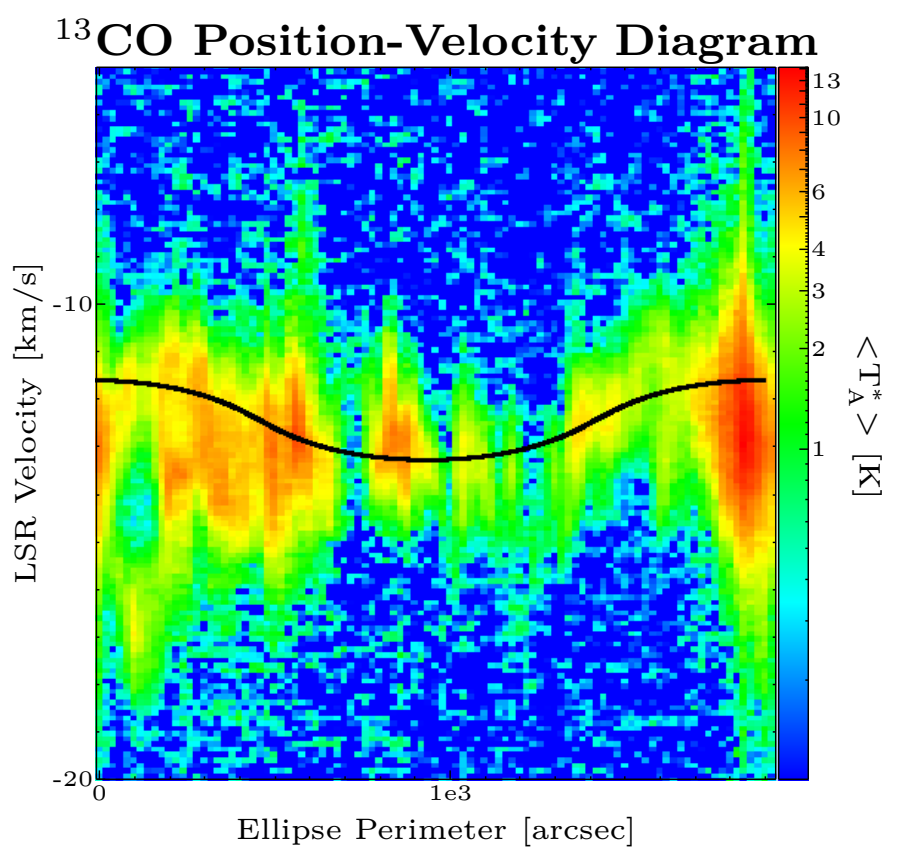

Fig. 12. Position-velocity diagram of the ${ }^{13} \mathrm{CO}(3-2)$ line along the elliptical curve of the expanding ring model in the $X-Y$ plane (see Fig. 2a); the black line shows the position-velocity diagram for the model in Sect. 3.11.

is $\sim 7 \times 10^{46} \mathrm{erg}\left(E=M V^{2} / 2\right)$. Table 4 summarizes these parameters, and Figs. $2 \mathrm{a}$ and 12 show the expanding ring model over the integrated velocity image and over the position-velocity diagram of the ${ }^{13} \mathrm{CO}$ line, respectively.

Figure $4 \mathrm{~d}$ shows the $35.6 \mathrm{~cm}$ continuum emission overlaid with the elliptical curve of the ring model in the $X-Y$ plane. The $35.6 \mathrm{~cm}$ source $\mathrm{J} 165920-400424$ is located within the expanding ring, at $\sim 30$ arcsec from its center, and without an infrared counterpart, as was discussed in Sect. 3.2. Thus it is possible that the expansion of the ring is caused by a supernova explosion, where J165920-400424 can be a pulsar (e.g. Whiteoak 1992), resulting 
Filament 1: ${ }^{13}$ CO P-V Diagram

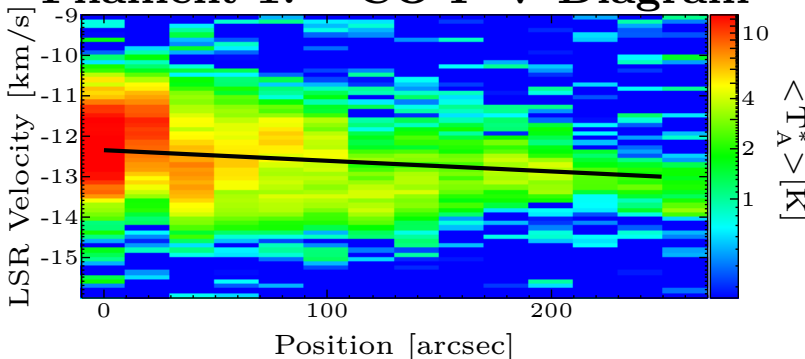

Filament 3: ${ }^{13}$ CO P-V Diagram

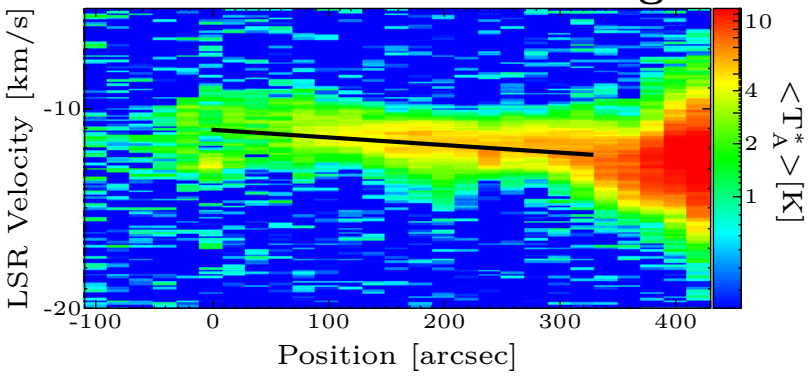

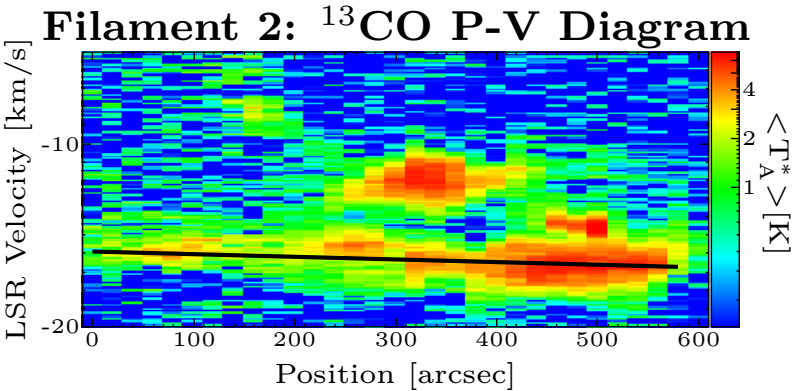

Filament 4: ${ }^{13} \mathrm{CO}$ P-V Diagram

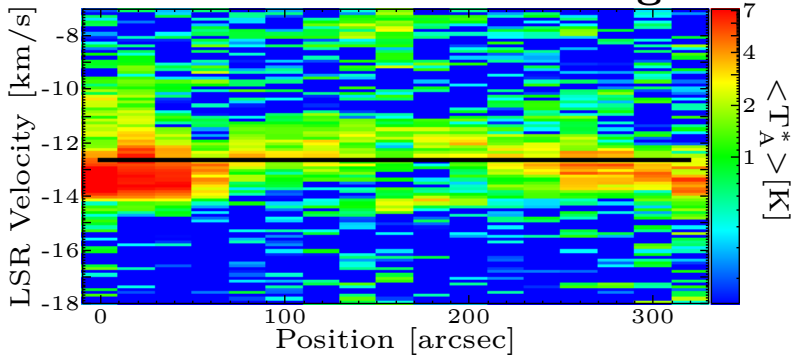

Filament 5: ${ }^{13}$ CO P-V Diagram

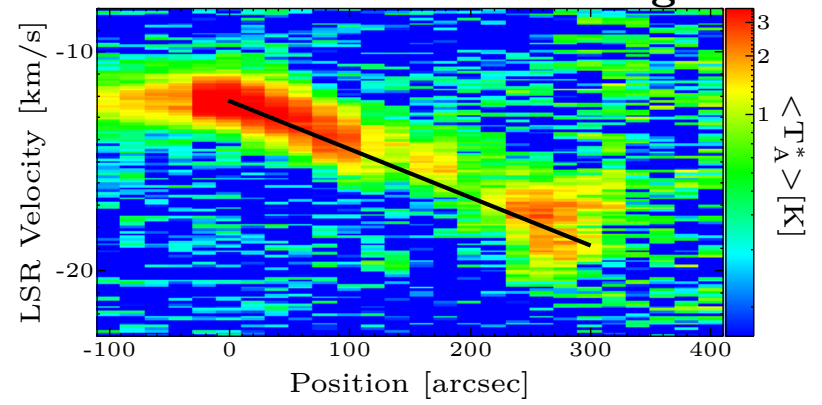

Fig. 13. ${ }^{13} \mathrm{CO}(3-2)$ line position-velocity diagrams for the five filaments. The black lines indicate the position-velocity diagrams of the filament models, see Sect. 3.13.

from the gravitational collapse of a massive star. This explanation needs to be tested in future observations (see Sect. 3.2).

\subsection{Molecular outflow in IRAS 16562-3959}

The outflow associated with the infrared source IRAS 165623959 (hereafter outflow IRAS 16562-3959) was first identified as a collimated jet by Guzmán et al. (2010) using 3.5-21 cm continuum emission. They determined an outflow size of $\sim 0.4 \mathrm{pc}$ (47" at $1.8 \mathrm{kpc})$.

In the ${ }^{12} \mathrm{CO}$ and ${ }^{13} \mathrm{CO}$ lines observations shown in Fig. 2e, this molecular outflow is identified by the broad line width and clear line wings.

From the ${ }^{12} \mathrm{CO}(3-2)$ line, the velocity range of the outflow is between -31.1 and $7.3 \mathrm{~km} \mathrm{~s}^{-1}$ with emission $>3 \sigma$, and centered at $-11.3 \mathrm{~km} \mathrm{~s}^{-1}$ calculated by fitting a Gaussian shape. Using the ${ }^{13} \mathrm{CO}(3-2)$ line and eliminating the ambient cloud component by fitting a Gaussian shape to the integrated spectrum of the central area of the clump within a radius of $23.5^{\prime \prime}$, the mass and projected energy of the outflow are $13 M_{\odot}$ and $7 \times 10^{45} \mathrm{erg}$, respectively.

Using the size determined from centimeter continuum emission by Guzmán et al. (2010), it is possible to calculate an expansion time of $10^{4} \mathrm{yr}$ and a mass outflow rate of $10^{-3} M_{\odot} \mathrm{yr}^{-1}$. Using the correlation luminosity-mass outflow rate estimated by Shepherd \& Churchwell (1996), the driving
Table 5. Characteristics of the outflow IRAS 16562-3959.

\begin{tabular}{ll}
\hline \hline Parameter & Value \\
\hline Spatial center $(\mathrm{J} 2000)$ & $16: 59: 41$ \\
& $-40: 03: 37$ \\
LSR velocity center $\left[\mathrm{km} \mathrm{s}^{-1}\right]$ & -11.3 \\
Projected size [pc] & $0.4^{\dagger}$ \\
Projected velocity range $\left[\mathrm{km} \mathrm{s}^{-1}\right]$ & 38.4 \\
Mass [ $M_{\odot}$ ] & 13 \\
Inclination [deg] & $41^{\dagger}$ \\
Energy [erg] & $7 \times 10^{45}$ \\
Expansion time [yr] & $10^{4 \ddagger}$ \\
Mass outflow rate $\left[M_{\odot} \mathrm{yr}^{-1}\right]$ & $2 \times 10^{-3} \ddagger$ \\
Luminosity [ $\left.L_{\odot}\right]$ & $5-6 \times 10^{4, \dagger, \star}$ \\
\hline
\end{tabular}

Notes. ${ }^{(\dagger)}$ From Guzmán et al. (2010). ${ }^{(\ddagger)}$ Using the size estimated by Guzmán et al. (2010). ${ }^{(\star)}$ From López et al. (2011).

source IRAS $16562-3959$ has to have a luminosity of $\sim 10^{3}-$ $10^{4} L_{\odot}$, which is comparable with the estimate obtained by integrating the spectral energy distribution (SED), $\sim 5-6 \times 10^{4} L_{\odot}$ (Guzmán et al. 2010; López et al. 2011).

All characteristics of this outflow are summarized in Table 5. 
Table 6. Characteristics of the filaments.

\begin{tabular}{lllllll}
\hline \hline Parameter & Filament 1 & Filament 2 & Filament 3 & Filament 4 & Filament 5 & Average \\
\hline Spatial Center $(\mathrm{J} 2000)$ & $16: 58: 56$ & $16: 59: 49$ & $16: 59: 54$ & $16: 58: 55$ & $16: 59: 13.79$ & \\
& $-40: 05: 36$ & $-40: 06: 00$ & $-39: 59: 34$ & $-40: 09: 36$ & $-39: 57: 27$ & \\
LSR velocity center $\left[\mathrm{km} \mathrm{s}^{-1}\right]$ & -12.7 & -16.3 & -11.7 & -12.6 & -15.5 & \\
Size $[\mathrm{pc}]$ & 2.2 & 5.1 & 2.9 & 2.8 & 2.6 & 3.1 \\
Projected velocity gradient $\left[\mathrm{km} \mathrm{s}^{-1} \mathrm{pc}^{-1}\right]$ & 0.3 & 0.2 & 0.5 & 0.0 & 2.5 & 0.7 \\
Spatial width $(F W H M)[\mathrm{pc}]$ & 0.35 & 0.67 & 0.65 & 0.51 & 0.93 & 0.62 \\
Velocity width $(F W H M)\left[\mathrm{km} \mathrm{s}^{-1}\right]$ & 2.5 & 2.1 & 2.6 & 2.2 & 2.5 & 2.4 \\
Mass $\left[10^{2} M_{\odot}\right]$ & 1 & 2.4 & 2.2 & 1.9 & 2.5 & 2.0 \\
Column density average $\left[10^{21} \mathrm{~cm}^{-2}\right]$ & 7.1 & 3.8 & 6.4 & 7.3 & 5.6 & 6.0 \\
\hline
\end{tabular}

\subsection{Filamentary structures}

At the edge of the ring, five filamentary structures are discovered, as indicated over the ${ }^{13} \mathrm{CO}$ line image shown in Fig. 2a. These structures are modeled as

$X(r)=X_{0}+r \cos (\theta)$

$Y(r)=Y_{0}+r \sin (\theta)$

$V_{\mathrm{z}}(r)=V_{0}+r \nabla V$,

where $r$ is the position in the filament, between $-S / 2$ and $S / 2$ with $S$ as the spatial size, $X(r)$ and $Y(r)$ are the projected spatial position, $\theta$ is the direction angle in the observed spatial plane $(X$ and $Y$ axes), $V_{z}(r)$ is the velocity in the $Z$ direction, $\nabla V$ is the projected velocity gradient, $X_{0}$ and $Y_{0}$ define the spatial center, and $V_{0}$ is the velocity center.

Using ${ }^{13} \mathrm{CO}$ emission, the sizes of these structures are of between 2.2 and $5.1 \mathrm{pc}$, their widths of between 0.35 and $0.93 \mathrm{pc}$, and their masses of between $\sim 100$ and $250 M_{\odot}$. Considering their masses, sizes, and spatial widths, their column density averages are between $3.8 \times 10^{21}$ and $7.3 \times 10^{21} \mathrm{~cm}^{-2}$. Almost all filaments show small velocity gradients $\left(<0.5 \mathrm{~km} \mathrm{~s}^{-1} \mathrm{pc}^{-1}\right)$, except for filament 5 , which has a velocity gradient of $2.5 \mathrm{~km} \mathrm{~s}^{-1} \mathrm{pc}^{-1}$. Table 6 summarizes the characteristics estimated for these filaments, and in Fig. 13 we show their ${ }^{13} \mathrm{CO}$ line position-velocity diagrams.

Since these filaments are not associated with infrared sources and almost all of them have small velocity gradients, they probably originate in the scenario where the low-density material in the molecular clouds first collapses into filamentary structures, which later fragment into clumps and cores, where the density reaches the critical level to form stars. This scenario has been outlined by observations toward regions of star formation (e.g. Molinari et al. 2010). For example, Goldsmith et al. (2008) found filaments of $0.8-1.0 \mathrm{pc}$ lenght with column densities of $\sim 10^{21} \mathrm{~cm}^{-2}$ in Taurus, which are similar to physical properties determined for the identified filaments here.

Using Herschel observations, several authors have determined a typical width of $\sim 0.1 \mathrm{pc}$ for the filaments in molecular clouds (e.g. Alves de Oliveira et al. 2014; Arzoumanian et al. 2011; Polychroni et al. 2013). Here, the widths of the filaments have an average of $\sim 0.6 \mathrm{pc}$, similar to other studies (e.g. Hennemann et al. 2012; Contreras et al. 2013), but this discrepancy might be caused by the lower spatial resolution in our observations.

\section{Conclusions}

The ring G345.45+1.50 was observed in the ${ }^{13} \mathrm{CO}(3-2)$ line. We list our conclusions below.
- The ring contains a total mass of $6.9 \times 10^{3} M_{\odot}$, which agrees well with results from previous observations in the $1.2 \mathrm{~mm}$ continuum emission.

- The ratio of the column density estimated from the $1.2 \mathrm{~mm}$ continuum to that estimated from the ${ }^{13} \mathrm{CO}(3-2)$ line varies between 0.1 and 10 , with an average value of $\sim 1.0$. The column density estimated from the $1.2 \mathrm{~mm}$ continuum is higher than that estimated from the ${ }^{13} \mathrm{CO}(3-2)$ line toward sites with high density. It is possible that the $1.2 \mathrm{~mm}$ continuum emission is more optically thin than the ${ }^{13} \mathrm{CO}(3-2)$ line toward dense regions.

- The ring is expanding with a velocity of $1.0 \mathrm{~km} \mathrm{~s}^{-1}$ and has an expansion timescale of $3 \times 10^{6} \mathrm{yr}$ and a total energy of $7 \times 10^{46} \mathrm{erg}$. This expansion might have been produced by a supernova explosion. This hypothesis is supported by the presence of a $35.6 \mathrm{~cm}$ source, J165920-400424, in the spatial center of the ring. This source does not have an infrared counterpart and might be a pulsar, that remained from the gravitational collapse of a massive star. This needs to be tested in future observations.

- From the ${ }^{13} \mathrm{CO}(3-2)$ line, the ring is composed of 104 clumps with diameters of between 0.3 and $1.6 \mathrm{pc}$, masses of between 2.3 and $7.5 \times 10^{2} M_{\odot}$, and densities of between $10^{2}$ and $10^{4} \mathrm{~cm}^{-3}$. About $6 \%$ of them show clear signs of star formation, which allowed us to estimate a typical lifetime for these clumps of $\lesssim 40 \mathrm{Myr}$.

- Assuming that clumps can be modeled as Bonnor-Ebert spheres, $\sim 13 \%$ of the clumps are gravitationally unstable, while most of the clumps require an external pressure with a median value of $4 \times 10^{4} \mathrm{~K} \mathrm{~cm}^{-3}$ to be in hydrostatic equilibrium.

- In the region, the outflow associated with the MSFR IRAS 16562-3959 was identified, with a velocity range of $38.4 \mathrm{~km} \mathrm{~s}^{-1}$, a mass of $13 M_{\odot}$, and a kinetic energy of $7 \times 10^{45} \mathrm{erg}$.

- At the edge of the ring, five filamentary structures are found with lengths of between 2.2 and $5.1 \mathrm{pc}$ and with masses of between $10^{2}$ and $2.5 \times 10^{2} M_{\odot}$.

Acknowledgements. C.L. acknowledges partial support from the GEMINICONICYT FUND, project No. 32070020, and ESO-University of Chile Student Fellowship. This work was supported by the Chilean Center for Astrophysics FONDAP No. 15010003 and by Center of Excellence in Astrophysics and Associated Technologies PFB 06. L.B. acknowledges support from CONICYT Project PFB06. I.d.G. acknowledges the Spanish MINECO grant AYA201130228-C03-01 (co-funded with FEDER fund). 


\section{References}

Alves, J., Lombardi, M., \& Lada, C. J. 2007, A\&A, 462, L17

Alves de Oliveira, C., Schneider, N., Merín, B., et al. 2014, A\&A, 568, A98

Anderson, L. D., Deharveng, L., Zavagno, A., et al. 2015, ApJ, 800, 101

Arzoumanian, D., André, P., Didelon, P., et al. 2011, A\&A, 529, L6

Bains, I., Wong, T., Cunningham, M., et al. 2006, MNRAS, 367, 1609

Beaumont, C. N., \& Williams, J. P. 2010, ApJ, 709, 791

Benjamin, R. A., Churchwell, E., Babler, B. L., et al. 2003, PASP, 115, 953

Bertoldi, F., \& McKee, C. F. 1992, ApJ, 395, 140

Blitz, L. 1993, in Protostars and Planets III, eds. E. H. Levy, \& J. I. Lunine (Tucson: University of Arizona Press), 125

Bloemen, J. B. G. M. 1987, ApJ, 322, 694

Bonnor, W. B. 1956, MNRAS, 116, 351

Bronfman, L., Alvarez, H., Cohen, R. S., \& Thaddeus, P. 1989, ApJS, 71, 481

Bronfman, L., Nyman, L.-A., \& May, J. 1996, A\&AS, 115, 81

Chavarría, L. A., Allen, L. E., Hora, J. L., Brunt, C. M., \& Fazio, G. G. 2008, ApJ, 682, 445

Churchwell, E. 2008, in Mass Loss from Stars and the Evolution of Stellar Clusters, eds. A. de Koter, L. J. Smith, \& L. B. F. M. Waters, ASP Conf. Ser., 388, 309

Churchwell, E., Povich, M. S., Allen, D., et al. 2006, ApJ, 649, 759

Churchwell, E., Watson, D. F., Povich, M. S., et al. 2007, ApJ, 670, 428

Contreras, Y., Rathborne, J., \& Garay, G. 2013, MNRAS, 433, 251

Faúndez, S., Bronfman, L., Garay, G., et al. 2004, A\&A, 426, 97

Frerking, M. A., Langer, W. D., \& Wilson, R. W. 1982, ApJ, 262, 590

Goldsmith, P. F., Heyer, M., Narayanan, G., et al. 2008, ApJ, 680, 428

Goodwin, S. P., Kroupa, P., Goodman, A., \& Burkert, A. 2007, Protostars and Planets V (Tucson: University of Arizona Press), 133

Goodwin, S. P., Nutter, D., Kroupa, P., Ward-Thompson, D., \& Whitworth, A. P. 2008, A\&A, 477, 823

Güsten, R., Nyman, L. A., Schilke, P., et al. 2006, A\&A, 454, L13

Guzmán, A. E., Garay, G., \& Brooks, K. J. 2010, ApJ, 725, 734

Heiles, C. 1979, ApJ, 229, 533

Hennemann, M., Motte, F., Schneider, N., et al. 2012, A\&A, 543, L3

Hildebrand, R. H. 1983, QJRAS, 24, 267

Kandori, R., Nakajima, Y., Tamura, M., et al. 2005, AJ, 130, 2166

Kawamura, A., Mizuno, Y., Minamidani, T., et al. 2009, ApJS, 184, 1

Kerton, C. R., Martin, P. G., Johnstone, D., \& Ballantyne, D. R. 2001, ApJ, 552, 601
Klessen, R. S. 2001, ApJ, 556, 837

Kramer, C., Stutzki, J., Rohrig, R., \& Corneliussen, U. 1998, A\&A, 329, 249

Kramer, C., Alves, J., Lada, C. J., et al. 1999, A\&A, 342, 257

Kroupa, P. 2002, Science, 295, 82

Kroupa, P. 2007, ArXiv Astrophysics e-prints [arXiv:astro-ph/0703124]

Lada, C. J., Alves, J. F., \& Lombardi, M. 2007, Protostars and Planets V (Tucson: University of Arizona Press), 3

López, C., Bronfman, L., Nyman, L.-Å., May, J., \& Garay, G. 2011, A\&A, 534, A131

Martín-Pintado, J., Gaume, R. A., Rodríguez-Fernández, N., de Vicente, P., \& Wilson, T. L. 1999, ApJ, 519, 667

Miura, R. E., Kohno, K., Tosaki, T., et al. 2012, ApJ, 761, 37

Molinari, S., Swinyard, B., Bally, J., et al. 2010, A\&A, 518, L100

Mookerjea, B., Kramer, C., Nielbock, M., \& Nyman, L.-Å. 2004, A\&A, 426, 119

Motte, F., Andre, P., \& Neri, R. 1998, A\&A, 336, 150

Muñoz, D. J., Mardones, D., Garay, G., et al. 2007, ApJ, 668, 906

Murphy, T., Mauch, T., Green, A., et al. 2007, MNRAS, 382, 382

Nutter, D., \& Ward-Thompson, D. 2007, MNRAS, 374, 1413

Oka, T., Hasegawa, T., Sato, F., Tsuboi, M., \& Miyazaki, A. 1998, ApJS, 118, 455

Ossenkopf, V., \& Henning, T. 1994, A\&A, 291, 943

Polychroni, D., Schisano, E., Elia, D., et al. 2013, ApJ, 777, L33

Price, S. D., Egan, M. P., Carey, S. J., Mizuno, D. R., \& Kuchar, T. A. 2001, AJ, 121,2819

Reid, M. A., \& Wilson, C. D. 2006, ApJ, 650, 970

Risacher, C., Vassilev, V., Monje, R., et al. 2006, A\&A, 454, L17

Schuller, F., Menten, K. M., Contreras, Y., et al. 2009, A\&A, 504, 415

Shepherd, D. S., \& Churchwell, E. 1996, ApJ, 472, 225

Sidorin, V., Douglas, K. A., Palouš, J., Wünsch, R., \& Ehlerová, S. 2014, A\&A, 565, A6

Tenorio-Tagle, G., \& Bodenheimer, P. 1988, ARA\&A, 26, 145

Tothill, N. F. H., White, G. J., Matthews, H. E., et al. 2002, ApJ, 580, 285

van Dishoeck, E. F. 2004, ARA\&A, 42, 119

Whiteoak, J. B. Z. 1992, A\&A, 262, 251

Williams, J. P., de Geus, E. J., \& Blitz, L. 1994, ApJ, 428, 693

Wilson, T. L., Rohlfs, K., \& Hüttemeister, S. 2009, Tools of Radio Astronomy (Springer-Verlag)

Wong, T., Ladd, E. F., Brisbin, D., et al. 2008, MNRAS, 386, 1069 


\section{Appendix A: LTE column density and mass estimated from the ${ }^{13} \mathrm{CO}(3-2)$ line}

Considering the rotational transition of a linear molecule from the upper level $\mathrm{u}$ to the lower level 1 , the column density of the lower level is (Wilson et al. 2009)

$N_{\mathrm{l}}=93.5 \mathrm{~cm}^{-2} \frac{g_{\mathrm{l}}(v / \mathrm{GHz})^{3}}{g_{\mathrm{u}}\left(A_{\mathrm{u} 1} / \mathrm{s}^{-1}\right)} \frac{1}{1-\exp \left(-h v / k T_{\mathrm{K}}\right)} \int \tau\left(\mathrm{d} v / \mathrm{km} \mathrm{s}^{-1}\right)$,

where $T_{\mathrm{ex}}$ is the excitation temperature, which is approximated to the kinetic temperature $T_{\mathrm{K}}, v$ is the frequency of transition, $\tau$ is the optical depth, $A_{\mathrm{u} 1}$ is the Einstein A coefficient of the radiative transition between the upper and lower levels, $v$ is the Doppler velocity, $g_{1}$ and $g_{u}$ are the statistical weights of the states. Assuming that $\tau \ll 1$ and the source observed fills the main beam, we approximate $T_{\mathrm{K}} \tau \sim T_{\mathrm{MB}} \sim T_{\mathrm{b}}$, where $T_{\mathrm{MB}}$ is the mainbeam brightness temperature and $T_{\mathrm{b}}$ is the brightness temperature. Thus

$$
\begin{aligned}
N_{1}= & 93.5 \mathrm{~cm}^{-2} \frac{g_{1}(v / \mathrm{GHz})^{3}}{g_{\mathrm{u}}\left(A_{\mathrm{u} 1} / \mathrm{s}^{-1}\right)} \\
& \times \frac{1}{1-\exp \left(-h v / k T_{\mathrm{K}}\right)} \frac{1}{T_{\mathrm{K}}} \int T_{\mathrm{b}}\left(\mathrm{d} v / \mathrm{km} \mathrm{s}^{-1}\right) .
\end{aligned}
$$

Assuming that all energy levels are populated under LTE conditions, the fraction of the total population in a particular state, $J$, is given by

$N(J) / N($ total $) \sim(2 J+1) \exp \left(-\frac{h B_{\mathrm{e}} J(J+1)}{k T_{\mathrm{K}}}\right) \frac{h B_{\mathrm{e}}}{k T_{\mathrm{K}}}$,

for $h B_{\mathrm{e}} \ll k T_{\mathrm{K}}$, where $B_{\mathrm{e}}$ is the rotational constant. Thus, the total density is given by

$$
\begin{aligned}
N(\text { total })= & 93.5 \mathrm{~cm}^{-2} \frac{g_{1}}{g_{\mathrm{u}}} \frac{(v / \mathrm{GHz})^{3}}{\left(A_{\mathrm{u} 1} / \mathrm{s}^{-1}\right)} \frac{k}{h B_{\mathrm{e}}} \frac{1}{2 J+1} \\
& \times \exp \left(\frac{h B_{\mathrm{e}}}{k} \frac{J(J+1)}{T_{\mathrm{K}}}\right) \frac{1}{1-\exp \left(-h v / k T_{\mathrm{K}}\right)} \\
& \times \int T_{\mathrm{B}}\left(\mathrm{d} v / \mathrm{km} \mathrm{s}^{-1}\right) .
\end{aligned}
$$

For the emission transition $J+1$ to $J, A_{\mathrm{u} 1}$ is given by

$A_{\mathrm{u} 1}=A_{\mathrm{J}}=1.165 \times 10^{-11} \mathrm{~s}^{-1}\left(\mu_{D} / \text { debye }\right)^{2}(v / \mathrm{GHz})^{3} \frac{J+1}{2 J+3}$,

$g_{1}=2 J+1$ and $g_{\mathrm{u}}=2 J+3$. Thus

$$
\begin{aligned}
N(\text { total })= & 80.3 \times 10^{11} \mathrm{~cm}^{-2} \frac{1}{\left(\mu_{D} / \text { debye }\right)^{2}} \frac{k}{h B_{\mathrm{e}}} \frac{1}{J+1} \\
& \times \exp \left(\frac{h B_{\mathrm{e}}}{k} \frac{J(J+1)}{T_{\mathrm{K}}}\right) \frac{1}{1-\exp \left(-h v / k T_{\mathrm{K}}\right)} \\
& \times \int T_{\mathrm{B}}\left(\mathrm{d} v / \mathrm{km} \mathrm{s}^{-1}\right) .
\end{aligned}
$$

For ${ }^{13} \mathrm{CO}$, the $B_{\mathrm{e}}$ is $5.5101 \times 10^{4} \mathrm{MHz}$, and $\mu_{D}, 0.11046$ debye ${ }^{7}$. Thus the total column density from the ${ }^{13} \mathrm{CO}(3-2)$ transition $(330.587960 \mathrm{GHz})$ is

$$
\begin{aligned}
N(\text { total })= & 8.30 \times 10^{13} \mathrm{~cm}^{-2} \frac{\exp \left(15.9 \mathrm{~K} / T_{\mathrm{K}}\right)}{1-\exp \left(-15.9 \mathrm{~K} T_{\mathrm{K}}\right)} \\
& \times \int T_{\mathrm{B}}\left(\mathrm{d} v / \mathrm{km} \mathrm{s}^{-1}\right),
\end{aligned}
$$

\footnotetext{
7 http://www.splatalogue.net/
}

where $T_{\mathrm{K}}$ is the kinetic temperature of gas, $\sim 30 \mathrm{~K}$ (see Sect. 3.3). The brightness temperature, $T_{\mathrm{b}}$, is estimated by Eq. (2) using $f_{\mathrm{BEAM}} \sim 1$ and $\eta_{\mathrm{MB}} \sim 0.73$.

Integrating Eq. (A.7) over the area, it is possible to derive a measurement of the mass

$$
\begin{aligned}
\frac{M^{13} \mathrm{CO}}{M_{\odot}}= & \int m_{\mathrm{H}} \mu N^{{ }^{13} \mathrm{CO}} \mathrm{d} A \sim 3.1 \times 10^{-11}\left[\frac{\mathrm{H}_{2}}{{ }^{13} \mathrm{CO}}\right] \frac{d^{2}}{\mathrm{kpc}} \\
& \times \alpha \frac{\exp \left(\frac{15.9 \mathrm{~K}}{T_{\text {ext }}}\right)}{1-\exp \left(\frac{-15.9 \mathrm{~K}}{T_{\text {ext }}}\right)} \int T_{\mathrm{B}} \frac{\mathrm{d} v}{\mathrm{~km} \mathrm{~s}^{-1}} \frac{\mathrm{d} \Omega}{\operatorname{arcsec}^{2}},
\end{aligned}
$$

where $\mu$ is the mean mass per particle, $\sim 2.29$ for an $\mathrm{H}_{2}$ cloud with a $25 \%$ of helium (Evans 1999), $\left[\mathrm{H}_{2} /{ }^{13} \mathrm{CO}\right]$ is the abundance ratio of $\mathrm{H}_{2}$ to ${ }^{13} \mathrm{CO}$ molecules, $\sim 7 \times 10^{5}$ (Frerking et al. 1982), $\alpha$ is the $\mathrm{H}_{2}$ mass correction to include helium mass, $\sim 1.3, \mathrm{~d} A$ is the differential area $\left(\mathrm{d} A=d^{2} \mathrm{~d} \Omega\right), d$ is the distance to the ring, $\sim 1.8 \mathrm{kpc}$, and $\Omega$ is the solid angle.

\section{Appendix B: Column density and mass estimated from $1.2 \mathrm{~mm}$ dust emission}

The column density can be estimated from the $1.2 \mathrm{~mm}$ dust continuum emission, $N_{1.2} \mathrm{~mm}$. This emission is assumed to be optically thin, thus the total column density is given by (Hildebrand 1983)

$N_{1.2 \mathrm{~mm}}=\frac{I_{1.2 \mathrm{~mm}}}{\mu m_{\mathrm{H}} k_{1.2 \mathrm{~mm}} B_{1.2 \mathrm{~mm}}\left(T_{\text {dust }}\right)} \frac{M_{\text {gas }}}{M_{\text {dust }}}$,

where $I_{1.2 \mathrm{~mm}}$ is the intensity, $m_{\mathrm{H}}$ is the hydrogen atom mass, $k_{1.2 \mathrm{~mm}}$ is the dust absorption coefficient, $\sim 1 \mathrm{~cm}^{2} \mathrm{~g}^{-1}$ for protostellar cores (Ossenkopf \& Henning 1994), $B_{1.2 \mathrm{~mm}}\left(T_{\text {dust }}\right)$ is the Planck function at dust temperature $T_{\text {dust }}, \sim 30 \mathrm{~K}$ for regions of massive star formation (Faúndez et al. 2004), and $M_{\mathrm{gas}} / M_{\mathrm{dust}}$ is the ratio of gas to dust masses, $\sim 100$ (Hildebrand 1983).

Integrating Eq. (B.1) over the area, it is possible to derive a measurement of the mass

$M_{1.2 \mathrm{~mm}}=\int m_{\mathrm{H}} \mu N_{1.2 \mathrm{~mm}} \mathrm{~d} A \sim \frac{S_{1.2 \mathrm{~mm}} d^{2}}{k_{1.2 \mathrm{~mm}} B_{1.2 \mathrm{~mm}}\left(T_{\text {dust }}\right)} \frac{M_{\mathrm{gas}}}{M_{\text {dust }}}$,

where $\mathrm{d} A$ is the differential area $\left(\mathrm{d} A=d^{2} \mathrm{~d} \Omega\right), d$ is the distance to the ring, $\sim 1.8 \mathrm{kpc}$, and $\Omega$ is the solid angle.

\section{Appendix C: Sizes and densities}

Clump diameters, $D_{\mathrm{c}}$, are estimated from the deconvolved FWHM size of their emissions, and calculated as

$D_{\mathrm{c}}=d \sqrt{\theta_{\mathrm{FWHM}}^{2}-\theta_{\text {beam }}^{2}}$,

where $\theta_{\text {bean }}$ is the beam-size, $\theta_{\text {FWHM }}$ is the observed FWHM and $d$ is the distance to the GMC.

Given the masses and diameters of clumps, the mean column density, $N_{\mathrm{c}}$, and the mean density, $\mathrm{n}_{\mathrm{c}}$, are calculated by

$N_{\mathrm{c}}=\frac{M_{\mathrm{c}}}{\pi\left(D_{\mathrm{c}} / 2\right)^{2}} \frac{1}{\mu m_{\mathrm{H}}}$

and

$n_{\mathrm{c}}=\frac{M_{\mathrm{c}}}{\frac{4}{3} \pi\left(D_{\mathrm{c}} / 2\right)^{3}} \frac{1}{\mu m_{\mathrm{H}}}$,

where $M_{\mathrm{c}}$ is the mass. 


\section{Appendix D: Virial equilibrium}

The virial condition is $2 T+W=0$, where $T$ is the kinetic energy and $W$ is the gravitational potential energy. For a clump with a homogeneous density distribution, this condition gives

$M_{\mathrm{vir}}=\frac{5 R_{\mathrm{c}} \sigma_{\mathrm{v}}^{2}}{G}$,

where $M_{\text {vir }}$ is the virial mass, $R_{\mathrm{c}}$ is the radius $\left(D_{\mathrm{c}} / 2\right)$, and $\sigma_{\mathrm{v}}$ is the velocity dispersion $\left(\sim \Delta V_{\mathrm{c}} / \sqrt{8 \ln (2)}\right)$. The parameter $\alpha_{\text {virial }}$ is defined as

$\alpha_{\text {virial }}=\frac{M_{\mathrm{vir}}}{M_{\mathrm{c}}}$,

where $M_{\mathrm{c}}$ is the mass of the clump.

\section{Appendix E: Bonnor-Ebert sphere}

The equation of hydrostatic equilibrium for a gas sphere is

$-\frac{\mathrm{d} p}{\mathrm{~d} r}=\frac{4 \pi G \rho}{r^{2}} \int_{0}^{r} \rho \bar{r}^{2} \mathrm{~d} \bar{r}$

where $p$ is the pressure, $r$ is the distance to the center, and $\rho$ is the density. Considering the equation of state $p=k \rho T_{\mathrm{K}} /\left(\mu m_{\mathrm{H}}\right)$, and making the following substitutions $\rho=\rho_{\mathrm{c}} \mathrm{e}^{-\psi}$ and $r=$ $\xi C_{\mathrm{s}} / \sqrt{4 \pi G}$, the equation of hydrostatic equilibrium becomes

$\frac{1}{\xi^{2}} \frac{\mathrm{d}}{\mathrm{d} \xi}\left(\xi^{2} \frac{\mathrm{d} \psi}{\xi}\right)=\mathrm{e}^{-\psi}$

where $\xi$ is a dimensionless variable, $\psi$ is a dimensionless function, $\rho_{\mathrm{c}}$ is the central density (at $r=0$ ), $C_{\mathrm{s}}$ is the isothermal sound speed, that is, $C_{\mathrm{s}}^{2}=k T_{\mathrm{k}} /\left(\mu m_{\mathrm{H}}\right)$ with $T_{\mathrm{K}}$ as the kinetic temperature.

Imposing the boundary conditions at $r=0: \rho(r)=\rho_{\mathrm{c}}$ and $\mathrm{d} \rho(r) / \mathrm{d} r=0$, that is, $\psi(\xi)=0$ and $\mathrm{d} \psi(\xi) / \mathrm{d} \xi$ at $\xi=0$, Eq. (E.2) is integrable numerically. If the gas sphere is confined by an external pressure $P_{\text {ext }}$ at the boundary defined by the Bonnor-Ebert radius $\left(R_{\mathrm{B}-\mathrm{E}}\right)$, the solution of Eq. (E.2) can be characterized by the dimensionless radius $\xi_{\max }=\xi\left(r=R_{\mathrm{B}-\mathrm{E}}\right)$, so $R_{\mathrm{B}-\mathrm{E}}$ can be expressed as

$R_{\mathrm{B}-\mathrm{E}}=\xi_{\mathrm{max}} C_{\mathrm{s}} / \sqrt{4 \pi G \rho_{\mathrm{c}}}$,

the external pressure as

$P_{\text {ext }}=\left.C_{\mathrm{s}}^{2} \rho_{\mathrm{c}} \mathrm{e}^{-\psi}\right|_{\xi_{\max }}$,

and the clump mass as

$M_{\mathrm{c}} \sim M_{\mathrm{BE}}=\left.\frac{C_{\mathrm{s}}^{2} R_{\mathrm{B}-\mathrm{E}}}{G} \xi_{\max } \frac{\mathrm{d} \psi}{\mathrm{d} \xi}\right|_{\xi_{\max }}$.

The stability of such a pressure-truncated gas spheres was investigated by Bonnor (1956), who showed that when $\xi_{\max }>6.5$ the spheres are in a state of unstable equilibrium, susceptible to gravitational collapse. Studies of the density profile toward isolated molecular clouds (Bok globules) in starless stages show that $\xi_{\max }$ is concentrated near the critical value $\left(\xi_{\max }=6.5\right)$, suggesting that this gaseous configuration represents the initial condition for star formation in dense cores (Lada et al. 2007).

Theoretically, the supporting mechanism in a Bonnor-Ebert sphere is purely the thermal pressure, but clumps also have nonthermal support (e.g., turbulence). The non-thermal part can be included in the model by replacing in Eq. (E.2) the isothermal sound speed $C_{\mathrm{s}}$ by an effective speed $C_{\text {eff }}$, where

$C_{\mathrm{eff}}^{2}=C_{\mathrm{s}}^{2}+C_{\mathrm{NT}}^{2}$

with $C_{\mathrm{NT}}$ as the non-thermal speed (e.g. Kandori et al. 2005).

The effective speed is approximated as

$C_{\text {eff }} \sim \frac{\Delta V_{\mathrm{c}}}{\sqrt{8 \ln (2)}}$

and the Bonnor-Ebert radius as

$R_{\mathrm{B}-\mathrm{E}} \sim \frac{D_{\mathrm{c}}}{2}$

where $D_{\mathrm{c}}$ and $\Delta V_{\mathrm{c}}$ are the diameter and line velocity width (FWHM) of the clumps, respectively. 


\section{Appendix F. Additionnal table}

Table F.1. Physical properties of the ${ }^{13} \mathrm{CO}$ clumps.

\begin{tabular}{|c|c|c|c|c|c|c|c|c|c|}
\hline Name & \multicolumn{2}{|c|}{$(\mathrm{J} 2000)$} & $\begin{array}{l}D_{\mathrm{c}} \\
\mathrm{pc}\end{array}$ & $\begin{array}{l}M_{\mathrm{c}} \\
M_{\odot}\end{array}$ & $\begin{array}{c}\Delta V_{\mathrm{c}} \\
\mathrm{km} \mathrm{s}^{-1}\end{array}$ & $\begin{array}{c}n_{\mathrm{c}} \\
\mathrm{cm}^{-3}\end{array}$ & $\begin{array}{c}N_{\mathrm{c}} \\
\mathrm{cm}^{-2}\end{array}$ & Clump type & B-E \\
\hline 1 & 16:59:06.92 & $-40: 13: 38.37$ & 1.0 & $2.3 \times 10^{2}$ & 2.0 & $8.7 \times 10^{3}$ & $1.7 \times 10^{22}$ & A & $\mathrm{Y}$ \\
\hline 2 & $16: 59: 41.33$ & $-40: 03: 46.92$ & 1.6 & $7.5 \times 10^{2}$ & 4.2 & $6.3 \times 10^{3}$ & $2.1 \times 10^{22}$ & A & $\mathrm{N}$ \\
\hline 3 & $16: 59: 45.58$ & $-40: 10: 16.90$ & 1.0 & $1.3 \times 10^{2}$ & 1.3 & $4.1 \times 10^{3}$ & $8.6 \times 10^{21}$ & $\mathrm{C}$ & $\mathrm{Y}$ \\
\hline 4 & $16: 59: 48.16$ & $-40: 10: 54.80$ & 1.3 & $2.4 \times 10^{2}$ & 1.2 & $3.4 \times 10^{3}$ & $9.5 \times 10^{21}$ & $\mathrm{C}$ & $\mathrm{Y}$ \\
\hline 5 & $16: 59: 07.18$ & $-40: 06: 05.01$ & 0.9 & $1.3 \times 10^{2}$ & 1.8 & $6.7 \times 10^{3}$ & $1.2 \times 10^{22}$ & B & $\mathrm{N}$ \\
\hline 6 & $16: 59: 11.91$ & $-40: 11: 05.78$ & 0.7 & $4.5 \times 10^{1}$ & 1.1 & $5.2 \times 10^{3}$ & $7.1 \times 10^{21}$ & B & $\mathrm{Y}$ \\
\hline 7 & $16: 59: 21.32$ & $-40: 11: 08.42$ & 1.0 & $1.2 \times 10^{2}$ & 1.5 & $4.4 \times 10^{3}$ & $8.9 \times 10^{21}$ & B & $\mathrm{Y}$ \\
\hline 8 & $16: 59: 08.19$ & $-40: 12: 54.83$ & 1.0 & $8.0 \times 10^{1}$ & 1.4 & $2.7 \times 10^{3}$ & $5.5 \times 10^{21}$ & $\mathrm{C}$ & $\mathrm{N}$ \\
\hline 9 & $16: 59: 49.55$ & $-40: 11: 46.04$ & 0.9 & $7.7 \times 10^{1}$ & 1.1 & $4.1 \times 10^{3}$ & $7.2 \times 10^{21}$ & $\mathrm{C}$ & $\mathrm{Y}$ \\
\hline 10 & $16: 59: 18.73$ & $-40: 10: 59.94$ & 1.0 & $4.0 \times 10^{1}$ & 0.8 & $1.4 \times 10^{3}$ & $2.8 \times 10^{21}$ & $\mathrm{~A}$ & $\mathrm{Y}$ \\
\hline 11 & $16: 59: 29.53$ & $-40: 10: 14.71$ & 1.2 & $1.3 \times 10^{2}$ & 1.4 & $2.4 \times 10^{3}$ & $6.0 \times 10^{21}$ & B & $\mathrm{Y}$ \\
\hline 12 & $16: 59: 34.55$ & $-40: 07: 36.79$ & 0.8 & $4.0 \times 10^{1}$ & 1.2 & $2.2 \times 10^{3}$ & $3.9 \times 10^{21}$ & B & $\mathrm{N}$ \\
\hline 13 & $16: 59: 04.56$ & $-40: 11: 24.80$ & 0.9 & $3.1 \times 10^{1}$ & 1.0 & $1.6 \times 10^{3}$ & $2.8 \times 10^{21}$ & B & $\mathrm{N}$ \\
\hline 14 & $16: 59: 25.25$ & $-40: 12: 52.81$ & 1.1 & $4.2 \times 10^{1}$ & 1.2 & $1.2 \times 10^{3}$ & $2.5 \times 10^{21}$ & $\mathrm{C}$ & $\mathrm{N}$ \\
\hline 15 & $16: 59: 15.70$ & $-40: 10: 00.09$ & 1.0 & $9.0 \times 10^{1}$ & 1.3 & $2.7 \times 10^{3}$ & $5.7 \times 10^{21}$ & $\mathrm{C}$ & $\mathrm{Y}$ \\
\hline 16 & $16: 59: 36.19$ & $-40: 12: 56.33$ & 0.4 & $3.1 \times 10^{1}$ & 2.6 & $1.4 \times 10^{4}$ & $1.2 \times 10^{22}$ & B & $\mathrm{N}$ \\
\hline 17 & $16: 59: 34.67$ & $-40: 09: 59.05$ & 1.4 & $1.5 \times 10^{2}$ & 1.3 & $1.8 \times 10^{3}$ & $5.2 \times 10^{21}$ & B & $\mathrm{Y}$ \\
\hline 18 & $16: 59: 53.37$ & $-40: 09: 04.01$ & 1.1 & $1.3 \times 10^{2}$ & 1.9 & $2.9 \times 10^{3}$ & $6.8 \times 10^{21}$ & B & $\mathrm{N}$ \\
\hline 19 & $16: 59: 37.24$ & $-40: 08: 32.24$ & 0.9 & $4.2 \times 10^{1}$ & 0.7 & $1.8 \times 10^{3}$ & $3.4 \times 10^{21}$ & $\mathrm{C}$ & Y \\
\hline 20 & $16: 59: 43.02$ & $-40: 08: 11.25$ & 1.4 & $1.3 \times 10^{2}$ & 1.4 & $1.6 \times 10^{3}$ & $4.6 \times 10^{21}$ & B & $\mathrm{N}$ \\
\hline 21 & $16: 59: 46.10$ & $-40: 09: 20.88$ & 1.1 & $7.3 \times 10^{1}$ & 1.4 & $2.0 \times 10^{3}$ & $4.4 \times 10^{21}$ & $\mathrm{C}$ & $\mathrm{N}$ \\
\hline 22 & $16: 59: 22.89$ & $-39: 58: 07.42$ & 0.8 & $5.0 \times 10^{1}$ & 1.6 & $2.9 \times 10^{3}$ & $5.0 \times 10^{21}$ & $\mathrm{C}$ & $\mathrm{N}$ \\
\hline 23 & $16: 59: 02.43$ & $-40: 04: 56.57$ & 1.1 & $4.4 \times 10^{1}$ & 1.6 & $1.2 \times 10^{3}$ & $2.6 \times 10^{21}$ & $\mathrm{C}$ & $\mathrm{N}$ \\
\hline 24 & $16: 59: 12.32$ & $-40: 09: 06.94$ & 0.8 & $2.8 \times 10^{1}$ & 1.1 & $1.9 \times 10^{3}$ & $3.1 \times 10^{21}$ & $\mathrm{C}$ & $\mathrm{N}$ \\
\hline 25 & $16: 59: 47.41$ & $-40: 02: 32.38$ & 1.0 & $6.4 \times 10^{1}$ & 1.7 & $2.0 \times 10^{3}$ & $4.3 \times 10^{21}$ & $\mathrm{C}$ & $\mathrm{N}$ \\
\hline 26 & $16: 59: 50.33$ & $-40: 00: 12.50$ & 1.1 & $4.7 \times 10^{1}$ & 1.0 & $1.3 \times 10^{3}$ & $2.8 \times 10^{21}$ & A & $\mathrm{N}$ \\
\hline 27 & $16: 59: 15.12$ & $-40: 00: 50.15$ & 0.6 & $1.2 \times 10^{1}$ & 1.2 & $1.6 \times 10^{3}$ & $2.1 \times 10^{21}$ & B & $\mathrm{N}$ \\
\hline 28 & $16: 59: 08.70$ & $-40: 02: 52.08$ & 0.7 & $3.6 \times 10^{1}$ & 2.0 & $3.2 \times 10^{3}$ & $4.8 \times 10^{21}$ & B & $\mathrm{N}$ \\
\hline 29 & $16: 59: 30.75$ & $-39: 57: 57.01$ & 0.8 & $2.9 \times 10^{1}$ & 0.8 & $1.8 \times 10^{3}$ & $3.0 \times 10^{21}$ & $\mathrm{C}$ & $\mathrm{N}$ \\
\hline 30 & $16: 59: 41.06$ & $-39: 58: 24.31$ & 0.9 & $4.4 \times 10^{1}$ & 1.2 & $2.1 \times 10^{3}$ & $3.9 \times 10^{21}$ & $\mathrm{~B}$ & $\mathrm{~N}$ \\
\hline 31 & $16: 59: 42.13$ & $-40: 10: 51.31$ & 1.2 & $3.8 \times 10^{1}$ & 1.1 & $8.5 \times 10^{2}$ & $2.0 \times 10^{21}$ & $\mathrm{~B}$ & $\mathrm{~N}$ \\
\hline 32 & $16: 59: 13.96$ & $-40: 13: 17.77$ & 1.2 & $5.7 \times 10^{1}$ & 1.4 & $1.2 \times 10^{3}$ & $3.0 \times 10^{21}$ & $\mathrm{C}$ & $\mathrm{N}$ \\
\hline 33 & $16: 59: 46.49$ & $-40: 02: 09.44$ & 1.5 & $1.3 \times 10^{2}$ & 2.1 & $1.4 \times 10^{3}$ & $4.3 \times 10^{21}$ & $\mathrm{C}$ & $\mathrm{N}$ \\
\hline 34 & $16: 59: 13.72$ & $-40: 14: 22.98$ & 0.8 & $2.9 \times 10^{1}$ & 1.2 & $1.7 \times 10^{3}$ & $2.9 \times 10^{21}$ & $\mathrm{C}$ & $\mathrm{N}$ \\
\hline 35 & $16: 59: 17.95$ & $-40: 11: 17.31$ & 1.3 & $5.7 \times 10^{1}$ & 1.2 & $8.6 \times 10^{2}$ & $2.3 \times 10^{21}$ & A & $\mathrm{N}$ \\
\hline 36 & $16: 59: 48.36$ & $-40: 08: 37.90$ & 0.8 & $2.2 \times 10^{1}$ & 1.5 & $1.6 \times 10^{3}$ & $2.5 \times 10^{21}$ & $\mathrm{C}$ & $\mathrm{N}$ \\
\hline 37 & $16: 59: 33.77$ & $-39: 56: 56.65$ & 0.8 & $2.2 \times 10^{1}$ & 0.9 & $1.5 \times 10^{3}$ & $2.4 \times 10^{21}$ & $\mathrm{C}$ & $\mathrm{N}$ \\
\hline 38 & 16:59:09.04 & $-40: 10: 23.56$ & 1.1 & $3.1 \times 10^{1}$ & 1.2 & $8.9 \times 10^{2}$ & $1.9 \times 10^{21}$ & $\mathrm{C}$ & $\mathrm{N}$ \\
\hline 39 & $16: 59: 05.79$ & $-40: 06: 35.22$ & 1.2 & $3.4 \times 10^{1}$ & 1.4 & $7.2 \times 10^{2}$ & $1.7 \times 10^{21}$ & $\mathrm{~B}$ & $\mathrm{~N}$ \\
\hline 40 & $16: 59: 28.09$ & $-39: 58: 06.82$ & 0.9 & $2.1 \times 10^{1}$ & 0.6 & $1.0 \times 10^{3}$ & $1.9 \times 10^{21}$ & B & $\mathrm{Y}$ \\
\hline 41 & $16: 59: 17.31$ & $-39: 58: 25.14$ & 0.8 & $1.6 \times 10^{1}$ & 1.4 & $1.1 \times 10^{3}$ & $1.8 \times 10^{21}$ & $\mathrm{C}$ & $\mathrm{N}$ \\
\hline 42 & $16: 59: 44.41$ & $-39: 59: 30.08$ & 0.8 & $2.5 \times 10^{1}$ & 1.2 & $1.7 \times 10^{3}$ & $2.8 \times 10^{21}$ & $\mathrm{~B}$ & $\mathrm{~N}$ \\
\hline 43 & $16: 58: 57.39$ & $-40: 05: 28.90$ & 1.1 & $3.0 \times 10^{1}$ & 1.5 & $8.3 \times 10^{2}$ & $1.8 \times 10^{21}$ & $\mathrm{C}$ & $\mathrm{N}$ \\
\hline 44 & $16: 59: 31.42$ & $-40: 06: 57.92$ & 0.9 & $5.3 \times 10^{1}$ & 1.3 & $2.2 \times 10^{3}$ & $4.2 \times 10^{21}$ & B & $\mathrm{N}$ \\
\hline 45 & $16: 59: 29.00$ & $-39: 56: 30.82$ & 0.8 & $1.5 \times 10^{1}$ & 0.6 & $9.0 \times 10^{2}$ & $1.5 \times 10^{21}$ & $\mathrm{C}$ & $\mathrm{N}$ \\
\hline 46 & $16: 59: 25.85$ & $-40: 13: 17.45$ & 0.7 & $1.2 \times 10^{1}$ & 1.3 & $1.1 \times 10^{3}$ & $1.6 \times 10^{21}$ & $\mathrm{C}$ & $\mathrm{N}$ \\
\hline 47 & $16: 59: 47.47$ & $-39: 59: 33.29$ & 1.1 & $4.8 \times 10^{1}$ & 1.0 & $1.3 \times 10^{3}$ & $2.8 \times 10^{21}$ & $\mathrm{C}$ & $\mathrm{N}$ \\
\hline
\end{tabular}

Notes. Column 1 gives names; Cols. 2 and 3, equatorial coordinates; Col. 4, diameters; Col. 5, masses; Col. 6, line velocity widths; Col. 7, densities; Col. 8, column densities; Col. 9, clump types; and Col. 10 indicates if clumps are candidates to be experimenting collapse ( $\xi_{\max } \geq 6.5$ ). 
Table F.1. continued.

\begin{tabular}{|c|c|c|c|c|c|c|c|c|c|}
\hline Name & \multicolumn{2}{|c|}{ (J2000) } & $\begin{array}{l}D_{\mathrm{c}} \\
\text { pc }\end{array}$ & $\begin{array}{l}M_{\mathrm{c}} \\
M_{\odot} \\
\end{array}$ & $\begin{array}{c}\Delta V_{\mathrm{c}} \\
\mathrm{km} \mathrm{s}^{-1}\end{array}$ & $\begin{array}{c}n_{\mathrm{c}} \\
\mathrm{cm}^{-3}\end{array}$ & $\begin{array}{c}N_{\mathrm{c}} \\
\mathrm{cm}^{-2}\end{array}$ & Clump type & $B-E$ \\
\hline 48 & $16: 59: 12.24$ & $-40: 02: 06.95$ & 0.5 & $1.1 \times 10^{1}$ & 0.9 & $2.7 \times 10^{3}$ & $2.9 \times 10^{21}$ & B & $\mathrm{N}$ \\
\hline 49 & $16: 59: 54.30$ & $-40: 13: 01.26$ & 0.7 & $1.6 \times 10^{1}$ & 1.6 & $1.8 \times 10^{3}$ & $2.5 \times 10^{21}$ & $\mathrm{C}$ & $\mathrm{N}$ \\
\hline 50 & $16: 59: 08.43$ & $-40: 09: 49.16$ & 0.8 & $1.3 \times 10^{1}$ & 0.9 & $8.9 \times 10^{2}$ & $1.5 \times 10^{21}$ & $\mathrm{C}$ & $\mathrm{N}$ \\
\hline 51 & $16: 58: 48.70$ & $-40: 08: 06.74$ & 0.6 & $1.5 \times 10^{1}$ & 1.0 & $2.0 \times 10^{3}$ & $2.5 \times 10^{21}$ & $\mathrm{C}$ & $\mathrm{N}$ \\
\hline 52 & $16: 58: 55.54$ & $-40: 08: 51.93$ & 0.6 & $6.5 \times 10^{0}$ & 0.9 & $1.1 \times 10^{3}$ & $1.3 \times 10^{21}$ & $\mathrm{C}$ & $\mathrm{N}$ \\
\hline 53 & $16: 59: 21.67$ & $-40: 09: 28.99$ & 0.9 & $2.9 \times 10^{1}$ & 1.0 & $1.4 \times 10^{3}$ & $2.6 \times 10^{21}$ & $\mathrm{C}$ & $\mathrm{N}$ \\
\hline 54 & $16: 59: 50.98$ & $-40: 03: 46.26$ & 1.0 & $2.9 \times 10^{1}$ & 1.8 & $1.1 \times 10^{3}$ & $2.2 \times 10^{21}$ & $\mathrm{C}$ & $\mathrm{N}$ \\
\hline 55 & 17:00:00.54 & $-40: 09: 38.67$ & 1.1 & $6.8 \times 10^{1}$ & 1.7 & $1.8 \times 10^{3}$ & $4.0 \times 10^{21}$ & B & $\mathrm{N}$ \\
\hline 56 & 16:59:09.15 & $-40: 01: 49.37$ & 0.7 & $8.1 \times 10^{0}$ & 0.6 & $1.0 \times 10^{3}$ & $1.3 \times 10^{21}$ & $\mathrm{C}$ & $\mathrm{N}$ \\
\hline 57 & $16: 59: 37.21$ & $-40: 07: 19.98$ & 1.3 & $2.6 \times 10^{1}$ & 1.0 & $4.4 \times 10^{2}$ & $1.1 \times 10^{21}$ & B & $\mathrm{N}$ \\
\hline 58 & $16: 59: 48.75$ & $-40: 05: 13.00$ & 1.3 & $2.8 \times 10^{1}$ & 1.1 & $4.3 \times 10^{2}$ & $1.1 \times 10^{21}$ & $\mathrm{C}$ & $\mathrm{N}$ \\
\hline 59 & $16: 59: 05.32$ & $-39: 56: 06.84$ & 0.5 & $1.4 \times 10^{1}$ & 1.9 & $4.2 \times 10^{3}$ & $4.1 \times 10^{21}$ & B & $\mathrm{N}$ \\
\hline 60 & $16: 59: 58.09$ & $-40: 08: 07.26$ & 1.0 & $3.4 \times 10^{1}$ & 1.2 & $1.0 \times 10^{3}$ & $2.2 \times 10^{21}$ & $\mathrm{C}$ & $\mathrm{N}$ \\
\hline 61 & $16: 59: 00.16$ & $-40: 10: 57.29$ & 0.7 & $9.2 \times 10^{0}$ & 0.7 & $7.6 \times 10^{2}$ & $1.2 \times 10^{21}$ & $\mathrm{C}$ & $\mathrm{N}$ \\
\hline 62 & $16: 59: 13.59$ & $-40: 10: 51.76$ & 1.3 & $1.5 \times 10^{1}$ & 1.0 & $2.2 \times 10^{2}$ & $6.0 \times 10^{20}$ & B & $\mathrm{N}$ \\
\hline 63 & $16: 59: 44.79$ & $-40: 07: 57.18$ & 1.1 & $3.3 \times 10^{1}$ & 0.8 & $9.4 \times 10^{2}$ & $2.1 \times 10^{21}$ & $\mathrm{C}$ & Y \\
\hline 64 & $16: 59: 41.38$ & $-39: 59: 31.70$ & 1.1 & $2.3 \times 10^{1}$ & 1.1 & $6.0 \times 10^{2}$ & $1.3 \times 10^{21}$ & B & $\mathrm{N}$ \\
\hline 65 & $16: 59: 02.30$ & $-40: 09: 45.35$ & 0.7 & $4.1 \times 10^{0}$ & 0.7 & $4.4 \times 10^{2}$ & $6.1 \times 10^{20}$ & $\mathrm{C}$ & $\mathrm{N}$ \\
\hline 66 & $16: 59: 31.41$ & $-40: 04: 52.24$ & 0.8 & $1.6 \times 10^{1}$ & 1.7 & $1.1 \times 10^{3}$ & $1.8 \times 10^{21}$ & $\mathrm{C}$ & $\mathrm{N}$ \\
\hline 67 & $16: 59: 05.65$ & $-39: 56: 18.12$ & 0.8 & $1.9 \times 10^{1}$ & 1.3 & $1.2 \times 10^{3}$ & $2.0 \times 10^{21}$ & B & $\mathrm{N}$ \\
\hline 68 & $16: 59: 29.06$ & $-39: 56: 49.57$ & 0.8 & $8.4 \times 10^{0}$ & 0.8 & $6.0 \times 10^{2}$ & $9.6 \times 10^{20}$ & $\mathrm{C}$ & $\mathrm{N}$ \\
\hline 69 & $16: 59: 45.98$ & $-39: 57: 07.56$ & 1.0 & $2.5 \times 10^{1}$ & 0.8 & $8.8 \times 10^{2}$ & $1.8 \times 10^{21}$ & $\mathrm{C}$ & $\mathrm{N}$ \\
\hline 70 & $16: 58: 51.12$ & $-40: 09: 22.29$ & 0.7 & $1.1 \times 10^{1}$ & 1.3 & $9.6 \times 10^{2}$ & $1.5 \times 10^{21}$ & $\mathrm{C}$ & $\mathrm{N}$ \\
\hline 71 & $16: 59: 49.75$ & $-40: 12: 19.65$ & 1.0 & $1.3 \times 10^{1}$ & 0.9 & $4.2 \times 10^{2}$ & $8.8 \times 10^{20}$ & $\mathrm{C}$ & $\mathrm{N}$ \\
\hline 72 & 17:00:01.15 & $-40: 10: 36.04$ & 1.2 & $8.4 \times 10^{0}$ & 0.8 & $1.8 \times 10^{2}$ & $4.3 \times 10^{20}$ & $\mathrm{C}$ & $\mathrm{N}$ \\
\hline 73 & $16: 59: 25.56$ & $-40: 14: 09.62$ & 0.8 & $3.4 \times 10^{0}$ & 0.4 & $2.4 \times 10^{2}$ & $3.8 \times 10^{20}$ & $\mathrm{C}$ & $\mathrm{N}$ \\
\hline 74 & 17:00:04.98 & $-40: 13: 51.41$ & 0.6 & $3.0 \times 10^{0}$ & 0.8 & $4.0 \times 10^{2}$ & $5.2 \times 10^{20}$ & $\mathrm{C}$ & $\mathrm{N}$ \\
\hline 75 & $16: 59: 53.42$ & $-40: 07: 18.93$ & 1.1 & $3.1 \times 10^{1}$ & 1.1 & $7.8 \times 10^{2}$ & $1.8 \times 10^{21}$ & $\mathrm{C}$ & $\mathrm{N}$ \\
\hline 76 & $16: 59: 50.12$ & $-39: 56: 08.24$ & 0.6 & $8.0 \times 10^{0}$ & 0.6 & $1.1 \times 10^{3}$ & $1.4 \times 10^{21}$ & $\mathrm{C}$ & $\mathrm{N}$ \\
\hline 77 & $16: 59: 54.21$ & $-39: 56: 12.17$ & 0.6 & $5.4 \times 10^{0}$ & 0.7 & $7.3 \times 10^{2}$ & $9.5 \times 10^{20}$ & $\mathrm{C}$ & $\mathrm{N}$ \\
\hline 78 & $16: 58: 56.16$ & $-40: 10: 17.33$ & 0.6 & $4.8 \times 10^{0}$ & 0.7 & $7.2 \times 10^{2}$ & $9.0 \times 10^{20}$ & $\mathrm{C}$ & $\mathrm{N}$ \\
\hline 79 & $16: 59: 55.60$ & $-39: 58: 21.95$ & 0.9 & $2.3 \times 10^{1}$ & 0.9 & $1.0 \times 10^{3}$ & $1.9 \times 10^{21}$ & B & $\mathrm{N}$ \\
\hline 80 & $16: 58: 54.33$ & $-40: 10: 02.60$ & 0.4 & $3.0 \times 10^{0}$ & 0.8 & $2.4 \times 10^{3}$ & $1.7 \times 10^{21}$ & $\mathrm{C}$ & $\mathrm{N}$ \\
\hline 81 & 17:00:12.15 & $-40: 11: 00.99$ & 0.7 & $4.7 \times 10^{0}$ & 1.5 & $4.2 \times 10^{2}$ & $6.3 \times 10^{20}$ & $\mathrm{C}$ & $\mathrm{N}$ \\
\hline 82 & $16: 59: 59.63$ & $-40: 06: 11.79$ & 0.9 & $9.5 \times 10^{0}$ & 1.5 & $4.2 \times 10^{2}$ & $7.9 \times 10^{20}$ & $\mathrm{C}$ & $\mathrm{N}$ \\
\hline 83 & $16: 59: 55.18$ & $-39: 58: 56.77$ & 0.9 & $1.7 \times 10^{1}$ & 0.7 & $7.4 \times 10^{2}$ & $1.4 \times 10^{21}$ & B & $\mathrm{N}$ \\
\hline 84 & $16: 59: 25.33$ & $-40: 08: 16.73$ & 0.7 & $1.1 \times 10^{1}$ & 0.9 & $1.3 \times 10^{3}$ & $1.7 \times 10^{21}$ & $\mathrm{C}$ & $\mathrm{N}$ \\
\hline 85 & $16: 59: 31.92$ & $-40: 09: 48.39$ & 1.2 & $1.4 \times 10^{1}$ & 0.9 & $2.6 \times 10^{2}$ & $6.4 \times 10^{20}$ & $\mathrm{C}$ & $\mathrm{N}$ \\
\hline 86 & $16: 59: 56.61$ & $-39: 56: 36.53$ & 0.7 & $7.9 \times 10^{0}$ & 0.9 & $9.7 \times 10^{2}$ & $1.3 \times 10^{21}$ & $\mathrm{C}$ & $\mathrm{N}$ \\
\hline 87 & 17:00:04.92 & $-39: 57: 44.23$ & 0.4 & $4.5 \times 10^{0}$ & 0.7 & $2.8 \times 10^{3}$ & $2.2 \times 10^{21}$ & $\mathrm{C}$ & $\mathrm{N}$ \\
\hline 88 & $16: 59: 54.33$ & $-40: 00: 52.24$ & 1.0 & $1.4 \times 10^{1}$ & 0.8 & $5.1 \times 10^{2}$ & $1.0 \times 10^{21}$ & $\mathrm{C}$ & $\mathrm{N}$ \\
\hline 89 & $16: 59: 41.43$ & $-39: 56: 45.98$ & 0.8 & $1.5 \times 10^{1}$ & 0.9 & $9.3 \times 10^{2}$ & $1.6 \times 10^{21}$ & $\mathrm{C}$ & $\mathrm{N}$ \\
\hline 90 & $16: 59: 26.35$ & $-40: 14: 22.66$ & 0.6 & $2.9 \times 10^{0}$ & 0.6 & $4.3 \times 10^{2}$ & $5.4 \times 10^{20}$ & $\mathrm{C}$ & $\mathrm{N}$ \\
\hline 91 & $16: 59: 58.63$ & $-39: 55: 16.58$ & 0.7 & $8.5 \times 10^{0}$ & 0.7 & $7.1 \times 10^{2}$ & $1.1 \times 10^{21}$ & $\mathrm{C}$ & $\mathrm{N}$ \\
\hline 92 & $16: 59: 43.34$ & $-40: 13: 25.33$ & 0.3 & $2.3 \times 10^{0}$ & 0.5 & $2.9 \times 10^{3}$ & $1.8 \times 10^{21}$ & $\mathrm{C}$ & $\mathrm{N}$ \\
\hline 93 & $16: 59: 58.94$ & $-39: 57: 00.07$ & 0.6 & $3.7 \times 10^{0}$ & 0.6 & $5.4 \times 10^{2}$ & $6.8 \times 10^{20}$ & $\mathrm{C}$ & $\mathrm{N}$ \\
\hline
\end{tabular}


Table F.1. continued.

\begin{tabular}{cccccccccc}
\hline \hline Name & RA & Dec & $\begin{array}{c}D_{\mathrm{c}} \\
\mathrm{pc}\end{array}$ & $\begin{array}{c}M_{\mathrm{c}} \\
M_{\odot}\end{array}$ & $\begin{array}{c}\Delta V_{\mathrm{c}} \\
\mathrm{km} \mathrm{s}^{-1}\end{array}$ & $\begin{array}{c}n_{\mathrm{c}} \\
\mathrm{cm}^{-3}\end{array}$ & $\begin{array}{c}N_{\mathrm{c}} \\
\mathrm{cm}^{-2}\end{array}$ & Clump type & B-E \\
\hline 94 & $16: 59: 43.79$ & $-40: 06: 24.61$ & 0.9 & $1.8 \times 10^{1}$ & 1.2 & $7.5 \times 10^{2}$ & $1.4 \times 10^{21}$ & $\mathrm{~A}$ & $\mathrm{~N}$ \\
95 & $16: 59: 46.42$ & $-39: 58: 02.25$ & 0.8 & $1.3 \times 10^{1}$ & 0.9 & $7.7 \times 10^{2}$ & $1.3 \times 10^{21}$ & $\mathrm{C}$ & $\mathrm{N}$ \\
96 & $16: 59: 51.38$ & $-39: 55: 57.85$ & 0.6 & $6.4 \times 10^{0}$ & 0.7 & $9.5 \times 10^{2}$ & $1.2 \times 10^{21}$ & $\mathrm{C}$ & $\mathrm{N}$ \\
97 & $17: 00: 01.61$ & $-40: 10: 06.77$ & 1.5 & $5.1 \times 10^{1}$ & 1.3 & $5.6 \times 10^{2}$ & $1.7 \times 10^{21}$ & $\mathrm{~B}$ & $\mathrm{~N}$ \\
98 & $17: 00: 01.71$ & $-39: 57: 22.28$ & 0.7 & $4.4 \times 10^{0}$ & 0.7 & $4.7 \times 10^{2}$ & $6.5 \times 10^{20}$ & $\mathrm{C}$ & $\mathrm{N}$ \\
99 & $16: 59: 41.32$ & $-39: 57: 09.75$ & 1.0 & $1.1 \times 10^{1}$ & 1.0 & $4.0 \times 10^{2}$ & $8.1 \times 10^{20}$ & $\mathrm{C}$ & $\mathrm{N}$ \\
100 & $17: 00: 01.60$ & $-40: 05: 21.98$ & 0.9 & $1.2 \times 10^{1}$ & 0.9 & $5.5 \times 10^{2}$ & $1.0 \times 10^{21}$ & $\mathrm{C}$ & $\mathrm{N}$ \\
101 & $16: 59: 33.24$ & $-40: 13: 18.61$ & 1.3 & $6.7 \times 10^{0}$ & 0.9 & $1.0 \times 10^{2}$ & $2.7 \times 10^{20}$ & $\mathrm{C}$ & $\mathrm{N}$ \\
102 & $17: 00: 09.38$ & $-39: 57: 04.29$ & 0.5 & $2.7 \times 10^{0}$ & 0.7 & $8.8 \times 10^{2}$ & $8.5 \times 10^{20}$ & $\mathrm{C}$ & $\mathrm{N}$ \\
103 & $16: 59: 54.25$ & $-40: 10: 14.04$ & 0.9 & $5.7 \times 10^{0}$ & 1.1 & $2.9 \times 10^{2}$ & $5.2 \times 10^{20}$ & $\mathrm{C}$ & $\mathrm{N}$ \\
104 & $16: 59: 47.17$ & $-40: 12: 36.94$ & 0.7 & $4.9 \times 10^{0}$ & 1.0 & $4.3 \times 10^{2}$ & $6.4 \times 10^{20}$ & $\mathrm{C}$ & $\mathrm{N}$ \\
\hline
\end{tabular}

